\title{
Phenotyping Polarization Dynamics Of Immune Cells Using A Lipid Droplet - Cell Pairing Microfluidic Platform
}

\author{
Léa Pinon ${ }^{1,2,3}$, Nicolas Ruyssen ${ }^{4}$, Judith Pineau ${ }^{2}$, Olivier Mesdjian ${ }^{1,3}$, Damien Cuvelier ${ }^{3,5,9}$, Rachele Allena ${ }^{6}$, Sophie \\ Asnacios $^{7,8}$, Atef Asnacios ${ }^{7}$, Paolo Pierobon ${ }^{2, 凶}$, and Jacques Fattaccioli ${ }^{1,3, \bowtie}$ \\ ${ }^{1}$ Laboratoire P.A.S.T.E.U.R., Département de Chimie, École Normale Supérieure, PSL Research University, Sorbonne Université, CNRS, 75005 Paris, France \\ ${ }^{2}$ Institut Curie, INSERM U932, Immunology and Cancer, INSERM, 75005 Paris, France \\ ${ }^{3}$ Institut Pierre-Gilles de Gennes pour la Microfluidique, 75005 Paris, France \\ ${ }^{4}$ Arts et Métiers Institute of Technology, Université Paris 13, Sorbonne Paris Cité, IBHGC, HESAM Université, F-75013 Paris, France \\ ${ }^{5}$ Institut Curie, PSL Research University, CNRS, UMR 144, Paris, France \\ ${ }^{6}$ LJAD, UMR CNRS 7351, Université Côte d'Azur, 06100, Nice, France \\ ${ }^{7}$ Université de Paris, CNRS, Laboratoire Matière et Systèmes Complexes, UMR 7057, F-75013, Paris, France \\ ${ }^{8}$ Sorbonne Université, Faculté des Sciences et Ingénierie, UFR 925 Physics, F-75005 Paris, France \\ ${ }^{9}$ Sorbonne Université, Faculté des Sciences et Ingénierie, UFR 926 Chemistry, F-75005 Paris, France
}

\begin{abstract}
The immune synapse is the tight contact zone between a lymphocyte and a cell presenting its cognate antigen. This structure serves as a signaling platform and entails a polarization of intra-cellular components, necessary to the immunological function of the cell. While the surface properties of the presenting cell are known to control the formation of the synapse, their impact on polarization has not yet been studied.

Using functional lipid droplets as tunable artificial presenting cells combined with a microfluidic pairing device, we simultaneously observe synchronized synapses and dynamically quantify polarization patterns of individual B cells. By assessing how the ligand concentration, the surface fluidity and the substrate rigidity impact this polarization, we show that its onset depends on the antigen concentration at the synapse, and that the substrate rigidity controls both its onset and its kinetics. Our experimental system enables a fine phenotyping of monoclonal cell populations based on their synaptic readout.
\end{abstract}

Oil-in-water droplets| B cell synapse| microfluidics| cell polarity| mechanosensitivity

Correspondence: paolo.pierobon@curie.fr and jacques.fattaccioli@ens.psl.eu

\section{Introduction}

Direct contact is an important channel of communication for cells in multicellular organisms. This is true for cells in tissues as well as for cells that mostly live as independent entities like immune cells (1). Their activation, their immune function and ultimately their fate depend on signal exchanges with other cells through an organized structure called immune synapse. In both B and T lymphocytes, the formation of the immune synapse is associated with a global rearrangement of the cytoskeleton and the establishment of a polarity axis (2, 3). B lymphocytes, the cells responsible for antibody production, encounter antigens in the subcapsular sinus of the lymph node, in soluble form or grafted on other cell surface such as macrophages or follicular dendritic cells. They recognize the antigen through their specific B cell receptor (BCR), internalize, process and further present it to a cognate $T$ cell. Antigen recognition entails membrane and intracellular reorganizations leading to the formation of the immune synapse (4). Engagement of the BCR leads to the clusterization of signaling complexes in a mechanosensitive way: signaling and size of the complexes depend on the rigidity of the substrate $(5,6)$. The synapse, in its final form, displays a stereotypical concentric shape with antigens/BCRs accumulated in a central cluster, surrounded by adhesion molecules and an actin ring at the periphery (7-9). A similar geometry is 
mirrored by cytoplasmic molecules close to the membrane (10). At the same time the microtubules network re-orient the traffic towards the synapse (polarization of the centrosome) to secrete lysosomal proteases and degrade the antigen in the synaptic cleft, in a process named enzymatic extraction (11). It has been shown that, depending on the deformability of the substrate, the antigen can be internalized also by mechanical pulling (12-15). When mechanical extraction fails, such as on non-deformable substrates, cells trigger the enzymatic extraction pathways described above (13). Interestingly, the mutual exclusivity of mechanical and enzymatic extractions suggests that the polarization mechanism is also sensitive to the mechanical properties of the substrate. Ultimately, the B cell immune synapse results in signal transduction, cell differentiation and production of high-affinity antibodies (2). It has been also proposed that by polarizing, B cells can divide asymmetrically to give rise to $B$ cells that present more efficiently the antigen to $T$ cells. Polarity, therefore, has consequences on B cell fate $(16,17)$.

All these experimental results point to a crucial role of physico-chemical properties of the antigen presenting surface in B cell activation. Different systems have been used to address this mechanism. For instance, clusters formation has been revealed on fluid interfaces allowing antigen mobility (lipid bilayers (7)); mechanosensitivity has been shown using deformable substrates such as soft gels $(5,6)$; antigen mechanical extraction has been uncovered on plasma membrane sheet (12), and quantified by calibrated DNA force sensors $(13,18)$. Despite the amount of information gathered in these systems, the heterogeneity of assays hinders the comparison between experiments and makes it impossible to evaluate the impact of independent properties on the synapse formation. This prompts us to introduce a new model to stimulate $B$ cells while independently controlling physical (rigidity, fluidity, size) and chemical (functionalization) properties: emulsion droplets.

Emulsions are colloidal liquid-liquid metastable suspensions stabilized by a surfactant monolayer, that have already shown their biocompatibility and their interest as probes when functionalized with proteins of interests in biophysical (19), developmental (20), and immunological contexts (21-24). By varying the bulk and surface composition, it is possible to tune the surface tension, hence the mechanical rigidity, independently from the ligand surface concentration, thus making lipid droplets a relevant antigen-presenting cell (APC) surrogate to stimulate B cells with the highest control on the physicochemical properties of the cognate surface.

In this work, we use this system to address the dependency of polarization on the physical and chemical properties of the antigen-presenting substrate. This has been neglected in the past, partially because of the heterogeneity of experimental models, partially because of the lack of reproducible way to study the global cell rearrangement following immune synapse formation over time. Therefore, for a proper quantitative study, we engineered antigen-functionalized lipid droplets with a pertinent set of physico-chemical properties and presented them to B cells in a controlled microfluidic pairing device that minimizes the stress to mimic the flow conditions of the lymph node. This allowed us to simultaneously observe multiple synchronized synapses with a high spatio-temporal resolution and finally provide a phenotyping map of variability of B cell polarity both in terms of polarization onset and kinetics. 


\section{Results}

\section{Droplets formulation and quantification of antigen concentration}

Our study has been conducted with B lymphoma murine cell line IIA1.6, which is described to be a homogenous population of non-activated mature B cells, lacking $\mathrm{Fc}_{\gamma} \mathrm{R}$ IIB1 receptors on the surface $(25,26)$ and hence can be conveniently activated through specific antibodies against the BCR, constituting an ideal model for functional studies of antigen presentation. We stimulated these cells with lipid droplets functionalized with such antibodies Fig. 1A.

Droplets fabrication. Lipid droplets are fabricated by shearing an oil phase in an aqueous buffer containing surface active agents (Poloxamer 188) to improve suspension stability over time (27). We obtain lipid droplets using either a Couettecell apparatus (28) or a SPG membrane emulsification (29) device, leading to, respectively, quasi-monodisperse and monodisperse size distributions (see Materials and Methods for details) with an average diameter of $11 \mu \mathrm{m}$ (Fig. 1B), comparable to the one of an APC. Monodispersity is a desired property since different curvatures of the presenting surfaces might introduce a bias in the analysis of synapse formation and cell polarization.

Droplet coating. Antigen recognition by BCRs is ensured with biotinylated $F\left(a b^{\prime}\right)_{2}$ fragments anchored on the droplet surface, which will be referred to as antigens $(\mathrm{Ag})$. Biotinylated phospholipids (DPSE-PEG 2000 -Biotin) are first inserted at the oil/water interface, followed by the specific adsorption of fluorescent streptavidin used as spacer between DPSE$\mathrm{PEG}_{2000}$-Biotin and biotinylated $\mathrm{F}(\mathrm{ab})_{2}$ fragments (Fig. 1A). As negative control, droplets are coated with biotinylated BSA which do not engage the BCR, in lieu of $F(a b)_{2}(15)$. To coat the droplet interface with phospholipids, we either dissolve the lipids in the oil prior to emulsification, (bulk-functionalization (22)), or adsorb the phospholipids at the surface after fabrication of the droplets (surface-functionalization, (30)). In this latter case, phospholipids are inserted to the oil/water interface with the help of a polar co-solvent, ensuring a homogeneous and finely-controlled lipid, and ultimately protein, surface concentration at the end of the adsorption process (Fig. 1C). The two protocols present different pros and cons that we will describe in the following.

Droplet function characterization. The surface-functionalization protocol ensures precise quantification of the amount of antigens displayed by droplets (30). We first titrate streptavidin attachment by measuring the adsorption isotherm onto the phospholipid-coated droplet surface (see Materials and Methods section for details). The bulk concentration of streptavidin is expressed in molar equivalents, i.e. the theoretical amount of proteins necessary to cover the droplet sample with a close packed monolayer as described in (30). For instance, one equivalent of streptavidin for 10 million of $11 \mu \mathrm{m}$-large droplets corresponds to $4.10^{-10}$ moles of proteins. The fluorescence of the droplets is characterized by epifluorescence microscopy, following the method detailed in Pinon et al. (30). The streptavidin adsorption isotherm fits with a Langmuir isotherm with a $\mathrm{K}_{S t r e p}$, the streptavidin concentration producing half occupation, equal to $9.7 \pm$ $4.7 \mathrm{eq}^{-1}$ compatible with previous analyses (Figure 1D)(30). For a bulk concentration of streptavidin corresponding to a maximal surface coverage (1 eq.), we measured the adsorption isotherm of antigens $\left(F\left(a b^{\prime}\right)_{2}\right)$ using fluorescent secondary antibodies and epifluorescence microscopy for the quantitative fluorescence measurements of the total amount of proteins 


\section{A}
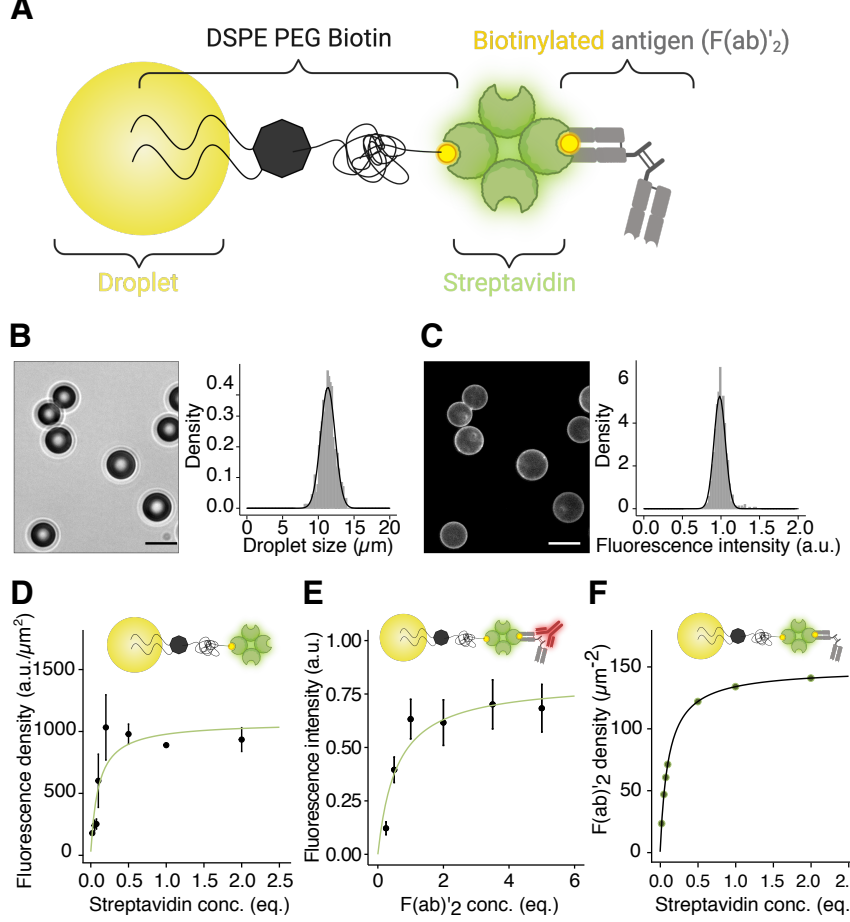

$\mathbf{F}$

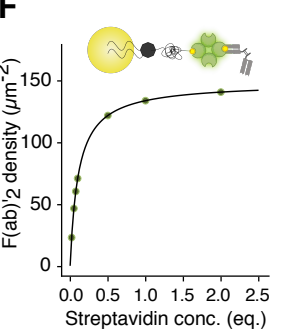

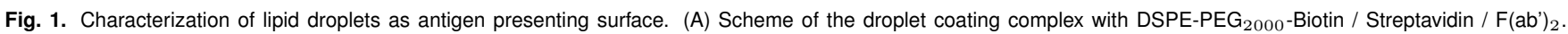
(B) Representative brightfield image of emulsion droplets and related size histogram, mean $=10.98 \pm 0.68 \mu \mathrm{m}$. Scale bar: $10 \mu \mathrm{m}$. (C) Representative epifluorescence image of coated droplet and related fluorescence histogram, mean $=1 \pm 0.075$ (normalized fluorescence). Channel: GFP, Scale bar: $10 \mu \mathrm{m}$. (D) Experimental titration curve of streptavidin on $11 \mu \mathrm{m}$ large droplets, initially coated with 100 equivalents of DSPE-PEG ${ }_{2000}$-biotin. (E) Experimental titration curve of $\mathrm{F}\left(\right.$ ab') ${ }_{2}$ fragments on $11 \mu \mathrm{m}$ large droplets, initially functionalized with 100 equivalents of DSPE-PEG 2000 -biotin and 1 equivalent of Streptavidin. Non-fluorescent $F(\text { ab') })_{2}$ fragments are imaged with a secondary fluorescent anti- $\left.\mathrm{F}(\mathrm{ab})_{2}\right)_{2}$ antibody, with a 1:1 ratio. $(\mathrm{F})$ Titration curve of the absolute value of the $\mathrm{F}(\mathrm{ab})_{2}$ fragments surface density on $11 \mu \mathrm{m}$ large droplets, initially functionalized with 100 equivalents of DSPE-PEG 2000 -biotin and 1 equivalent of streptavidin. The surface density spans over 2 orders of magnitude.

attached to droplets. Figure 1E shows that the antigen adsorption isotherm fits with a Langmuir isotherm with a $\mathrm{K}_{A g}=$ $1.74 \pm 0.74 \mathrm{eq}^{-1}$. We finally converted the streptavidin fluorescence intensity value in a molecular equivalent of proteins using fluorescence calibration beads, and knowing the average number of dyes attached per streptavidin (and therefore per $\mathrm{F}(\mathrm{ab})_{2}$, Fig. S1). Figure 1F shows that lipid droplets present up to 150 antigens/ $\mu \mathrm{m}^{2}$ at saturating conditions corresponding to the antigen range presented by other systems to activate B cells (8). Following this calibration, bulkfunctionalized droplets (22) are measured to intrinsically bear 50 antigens $/ \mu \mathrm{m}^{2}$.

\section{Droplet mechanical properties mimic antigen-presenting cells ones}

Antigen-presenting cells stiffness has been reported to be a relevant mechanical property involved in the synapse formation, the immune cell polarization and B cell activation $(5,31,32)$. Oil-droplets as surrogate APCs should display the same range of stiffness as APC even though they are not expected to mimic the complex mechanical behaviour of a cell. The stiffness of a material is related to its ability to resist reversible deformations when submitted to stress, i.e. to its apparent elastic properties. For oil droplets, the origin of such an elastic-like resistance to deformation is expected to be the excess pressure inside the droplet, i.e. the Laplace pressure $\Delta \mathrm{P}$ which is proportional to the interfacial tension $\gamma$, between the droplet and the surrounding liquid medium, and scales as the inverse of the droplet radius $\mathrm{R}$ as $\Delta \mathrm{P}=2 \gamma / \mathrm{R}$. The stiffness of a droplet could, therefore, be modulated by the oil and its surface tension $\gamma$ with the surrounding liquid medium. 
Characterization by pendant drop. We thus selected a range of droplet formulations characterized by different surface tensions, which we carefully measured using the pendant drop technique $(33,34)$. This technique consists in inferring the interfacial tension from the shape profile of a pendant drop of one liquid in another at mechanical equilibrium. In our case, the drop is made from one of the selected oils: (1) soybean oil, (2) mineral oil, (3) mineral oil + oleic acid, (4) an halogenated vegetable oil (lipiodol$($ ) $)$, and (5) lipiodol + oleic acid; and the aqueous phase is composed of water and surfactants, namely F68 and Tween 20. Table 1 shows that the interfacial tension of the oil/water interface ranges from about 1 to $12 \mathrm{mN} / \mathrm{m}$, thus varying by about one order of magnitude between the softest and stiffest formulations.

\begin{tabular}{|c|c|c|}
\hline Oil phase & Water Phase & Surface tension $(\mathbf{m N} / \mathbf{m})$ \\
\hline Mineral oil + Oleic acid 5\%v/v & F68 15\% & $1.69 \pm 0.19$ \\
\hline Mineral oil & F68 15\% & $4.73 \pm 0.38$ \\
\hline Soybean oil & F68 15\% & $12.03 \pm 0.52$ \\
\hline Lipiodol oil & F68 15\% & $1.52 \pm 0.12$ \\
\hline Lipiodol oil + Oleic acid 5\%v/v & F68 15\% & $0.88 \pm 0.12$ \\
\hline
\end{tabular}

Table 1. Interfacial tension of oil/aqueous phases via pendant drop technique.

Static characterization by micropipette aspiration. To better evaluate the effect of the lipid functionalization on the oil/water interfacial tension, we used another method, the micropipette aspiration technique. This involves the manipulation of individual emulsion droplets using equally small suction pipettes (see Materials and Methods). The interfacial tension is quantified by applying Laplace law upon aspiration of the droplets and measuring the curvature radii of the droplet both outside and inside the pipette. Due to the configuration of the experiment, we restricted this measurement to lipiodol droplets whose oil is denser than water, with or without biotinylated lipids (100 eq.). Table 2 shows that phospholipid functionalization does not change the oil/water surface tension. By comparing the value of surface tension of droplets having the same composition in Table 1, we see that the values obtained via the micropipette technique are slightly higher than the ones measured using the pendant drop technique, while remaining of the same order of magnitude (35).

\begin{tabular}{|c|c|c|c|}
\hline Oil phase & Surfactants & Functionalization & Surface tension $(\mathbf{m N} / \mathbf{m})$ \\
\hline Lipiodol oil + Oleic acid 5\%v/v & F68 - Tween 20 & None & $2.36 \pm 0.31$ \\
\hline Lipiodol oil + Oleic acid 5\%v/v & F68 - Tween 20 & DSPE-PEG-Biotin & $2.50 \pm 0.24$ \\
\hline
\end{tabular}

Table 2. Interfacial tension of oil/aqueous phases via micropipette technique.

Dynamic characterization by microplates. Cell mechanics is usually characterized by both elastic and viscous behaviours (36). We measured, therefore, the viscoelastic modulus of single droplets with a microplates-based rheometer already used for myeloid APC $(31,37)$, which also enabled us to correlate the droplets interfacial tension with their storage modulus. The experiment consisted in (i) trapping a single droplet between two glass microplates, a rigid and a flexible one, (ii) applying a sinusoidal deformation while measuring the resulting amplitude and phase shift at the tip of the flexible microplate (Fig. 2A, B). From the applied oscillatory normal stress and the sinusoidal strain (deformation) of the droplet, one can infer the droplet complex dynamic modulus $\mathrm{G}^{*}(\mathrm{f})=\mathrm{G}^{\prime}+i \mathrm{G}^{\prime \prime}$, with the real part $\mathrm{G}^{\prime}$ representing the storage modulus (elastic-like response), and the imaginary part $\mathrm{G}^{\prime \prime}$ accounting for energy dissipation (viscous-like response).

Lipidiol droplets exhibited a Kelvin-Voigt behavior $\left(\mathrm{G}^{\prime}=\right.$ constant and $\mathrm{G}^{\prime \prime}$ proportional to the frequency (Fig. 2C and 
Supplementary Note 3) with a dominant elastic-like response $\left(G^{\prime} \gg G^{\prime \prime}\right.$, Supplementary Note 3). Moreover, we found that, for a fixed strain, the droplet storage modulus decreases as the inverse of the diameter of the resting droplet (Fig. 2D), in agreement with the assumption that the only restoring force resisting compression originates from Laplace pressure $\Delta P=4 \gamma / L_{0}$ (Supplementary Note 3), with $\gamma$ the surface tension of the droplet, and $L_{0}$ its resting diameter. Thus, by fitting of the storage modulus as function of the droplet diameter, we get an estimation of the droplet surface tension, $\gamma=1.21 \pm 0.04 \mathrm{mN} / \mathrm{m}$ (Fig. 2D and Supplementary Note 3), a value compatible with the one measured by the static methods described above (see Table 2).

As for micropipette experiments, only lipiodol droplets could be mechanically tested (see Materials and Methods). As all droplets are made of similar materials (oil, water and surfactants), we expect all kind of droplets to follow Laplace law, including those having a low oil density and small sizes (lower than $11 \mu \mathrm{m}$ ) for which deformations are below the resolution limit. Hence, static surface tensions of $1.7,4.7$ and $12 \mathrm{mN} / \mathrm{m}$ (for $11 \mu \mathrm{m}$-large droplets), correspond to elastic moduli of respectively 4.0, 11.7 and $29.8 \mathrm{kPa}$. Remarkably, for the measured resting diameters (from 15 to $54 \mu \mathrm{m}$ ), lipiodol droplets have storage moduli ranging from $500 \mathrm{~Pa}$ to $1500 \mathrm{~Pa}$ (Fig. 2D), in the same range as those reported for human APC (31) and in the range of the substrate rigidity capable of eliciting a strong B-cell signal according to (5).

Characterization of B cell mechanics. We also investigated the B lymphocyte apparent rigidity to compare their stiffness to the droplet one. We performed the same microplate experiments as described above on B cells (Fig. 2E). The results show that B cell mechanical properties follow a damping model (Fig. 2F). G' and $G^{\prime \prime}$ mainly behave as weak power laws of the frequency $(38,39)(\mathbf{F i g} . \mathbf{2} \mathbf{F})$ with a power law exponent similar to those already reported for immune cells $(\alpha \approx 0.16$, Fig. S3), and exhibit an apparent visco-elastic modulus of about $165 \mathrm{~Pa}$ (Fig. 2G). Hence, B cells are 3-fold softer than live dendritic cells and 6-fold than macrophages. These results confirm that B cells face in vivo APC generally stiffer than themselves, condition that we will reproduce in our system.

In the following part of the manuscript, we will stimulate B cells with droplets of different rigidities by changing the surface tension as described above, while keeping the size of the particles constant and similar to an APC size (diameter of 11 $\mu \mathrm{m})$.

\section{A microfluidic pairing device that respects the low shear-stress conditions of a lymphoid tissue}

Our next goal is to recreate conditions approaching the in vivo micro-environment where B cells form immune synapses. We designed a microfluidic chip to force single cell-droplet interaction and allow the simultaneous imaging of multiple synapses with a high temporal and spatial resolution (Fig. 3A and B). The microfluidic chip, inspired by (40), was engineered to apply the least perturbative shear stress as possible, mimicking the in vivo-like shear stress estimated in the subcapsular sinus lumen of lymphoid tissues (41). It consists of double-layered U-shaped traps where droplets and cells are sequentially trapped (Fig. $\mathbf{3 C}$ ). The structure has been dimensioned to trap cells and droplets of the same size: the whole chamber is composed of ca. 200 double-layered structures with $5 \mu \mathrm{m}$-thick, $25 \mu \mathrm{m}$-long and $5 \mu \mathrm{m}$-large pillars on $10 \mu \mathrm{m}$-thick, $12 \mu \mathrm{m}$-large and $30 \mu \mathrm{m}$-long U-shaped traps. Double layer structures result in fluid streamlines not being deviated when a first object is trapped, thus allowing the easy capture of a second object (40). In addition to this, fluid 
A

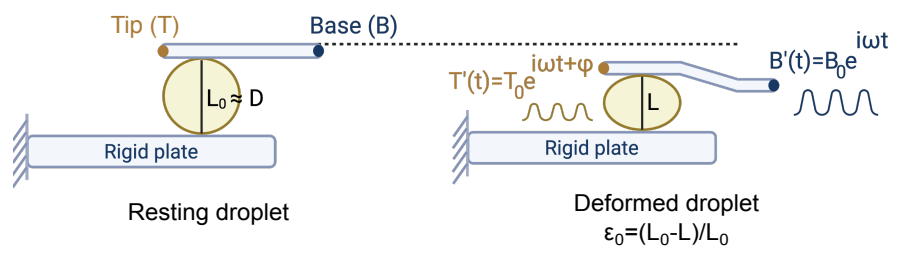

C

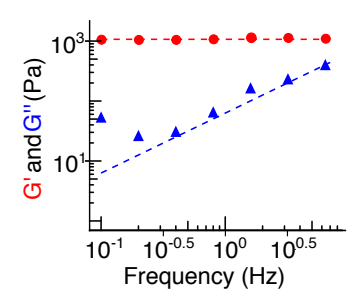

D

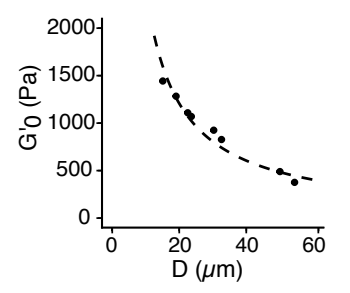

E

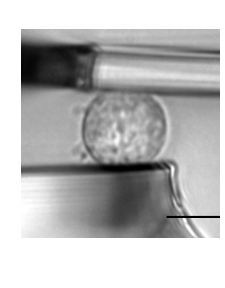

B

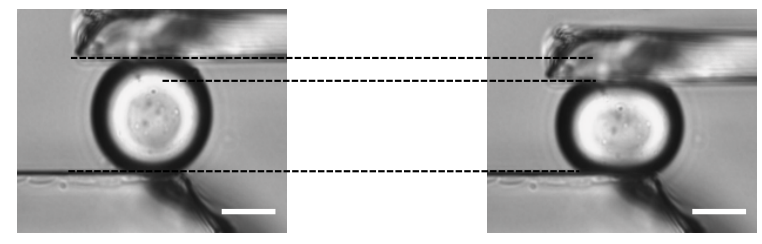

F

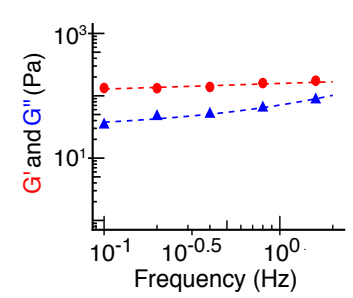

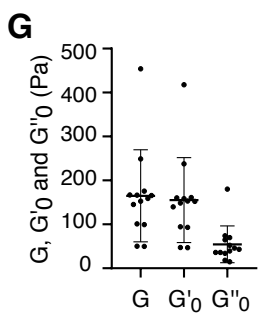

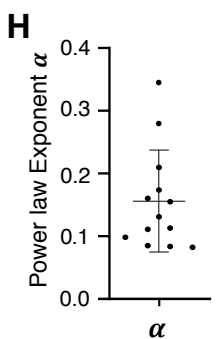

Fig. 2. Determination of mechanical properties of droplets and B cells. (A) Schematic representation of the microplates experiments protocol. A cell or a droplet is trapped between two glass microplates, one being immobile (bottom) the other flexible (top) with a base position oscillating as $\mathrm{B}(t)=B_{0} e^{i \omega t}$. We measure the oscillating displacement of the tip of the flexible plate as $\mathrm{T}(t)=\mathrm{T}_{0} e^{(i \omega t+\phi)}$ that relates to the immobilized cell or droplet visco-elastic properties. $\mathrm{T}$ is determined for a frequency range between $0.1 \mathrm{~Hz}$ and $6.4 \mathrm{~Hz}$ (droplet) or $1.6 \mathrm{~Hz}$ (cells). (B) Representative brightfield images of a resting (left) and a deformed (right) droplet. Scale bars: $30 \mu \mathrm{m}$. (C) Elastic G' and viscous $\mathrm{G}^{\prime \prime}$ moduli of a single droplet (diameter: $19.1 \mu \mathrm{m}$ ) as a function of the probing frequency. In this frequency range, $G^{\prime}$ is constant, whereas $\mathrm{G}^{\prime \prime}$ linearly depends on frequency. (D) Elastic modulus of droplets plotted as a function of the resting droplet diameter, for a constant initial deformation $\epsilon_{0}=0.2$. The fitting equation is written as $\mathrm{G}^{\prime}=4 \gamma / \epsilon \mathrm{L}_{0}$ and leads to a surface tension $\gamma=1.21 \pm 0.04 \mathrm{mN} \cdot \mathrm{m}^{-1}$.(E) Representative brightfield image of a B cell immobilized between the microplates. Scale bar: 10 $\mu \mathrm{m}(\mathrm{F})$ Elastic $G^{\prime}$ and viscous $\mathrm{G}^{\prime \prime}$ modulus of a single B cell plotted as a function of the probing frequency. Both $\mathrm{G}^{\prime}$ and $\mathrm{G}^{\prime \prime}$ follow a power law. ( $\mathrm{G}$ ) Summary graph of the $\mathrm{G}^{\prime}, \mathrm{G}^{\prime \prime}$ and $\mathrm{G}^{*}$ values of the $\mathrm{B}$ cells probed with the microplates $\left(\mathrm{N}=17\right.$ cells, $\mathrm{n}=3$ independent experiments). The average values of $\mathrm{G}_{0}^{*}$, $\mathrm{G}_{0}^{\prime}$ and $\mathrm{G}_{0}^{\prime \prime}$ are respectively equal to $165.0 \pm 57.0 \mathrm{~Pa}, 155.4 \pm 52.54 \mathrm{~Pa}$, and $54.5 \pm 22.7 \mathrm{~Pa}$. $(\mathrm{H})$ For $\mathrm{B}$ cells, the complex modulus $\mathrm{G}$ follows a power law of the frequency with coefficient $\alpha$ : $\mathrm{G}^{\prime}=\mathrm{G}_{0}^{\prime} f^{\alpha}$ and $\mathrm{G}^{\prime \prime}=\mathrm{G}_{0}^{\prime \prime} f^{\alpha}$. The exponent is determined from fitting of both moduli: $\alpha=0.155 \pm 0.04$ ( $\mathrm{N}=13, \mathrm{n}=3$ independent experiments), corresponding to values measured for immune cells.

flows are uniformly distributed across the chip, suggesting that all trapped cells are exposed to the same range of stresses in the whole chip (Fig. 3D).

Finite element simulations indicate that, at a maximum inlet fluid velocity of $1.6 \mathrm{~mm} / \mathrm{s}$, the maximal wall (on pillars) shear stress in the traps is $0.56 \mathrm{~Pa}$ (Fig. 3E, Fig. S4A and B). A 3D analysis has been performed to compute the shear stress that a single cell experiences when it is trapped in a weir structure (Fig. 3F). For the same maximal conditions (fluid velocity $=1.6 \mathrm{~mm} / \mathrm{s}$ ), a trapped cell is subjected to a shear stress of between 0.7 and 1.0 Pa. These values are compatible with the shear stress estimated in the lymph node (41). Of note, these conditions also ensure droplet stability all along the experiment (Fig. S5). Overall, the microfluidic chip we designed minimizes the stress to mimic conditions that a cell experiences in lymph nodes.

\section{B cell polarization triggering and dynamics}

Using the microfluidic chip described above, we formed doublets of IIA1.6 B cells and droplets coated with anti-lgG mouse $\mathrm{F}(\mathrm{ab})_{2}$, and imaged cell-droplet encounters from time zero to $40 \mathrm{~min}$ after antigen recognition. As a proxy for cell polarization, we followed lysosomes distribution in time, imaging them using an acidic compartment dye (Lysotracker®) over $40 \mathrm{~min}$ (Fig. 4A). This method is fast, reliable and does not interfere with organelles and cytoskeleton dynamics (11). Asymmetry in lysosomes distribution (polarity) is quantified by a polarization index $\mathrm{I}_{\text {pol }}$, defined as the ratio between the fluorescence signal at the front (the half cell in contact with the droplet) and the back of the cell following the droplet-cell axis (Fig. 4B). To establish the value of $\mathrm{I}_{p o l}$ for which we consider a cell to be polarized, we observe the $\mathrm{I}_{p o l}$ distribution for cells in contact with the non-activating droplet (BSA-coated, Fig. 4C). As spontaneous polarization has been reported 
A

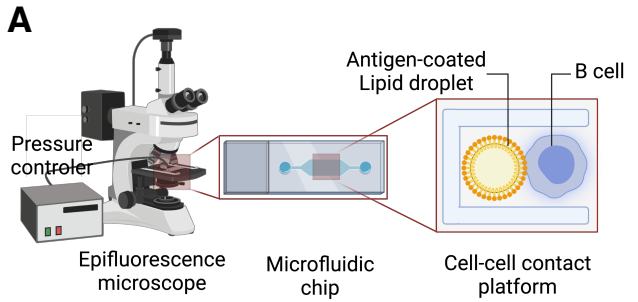

D

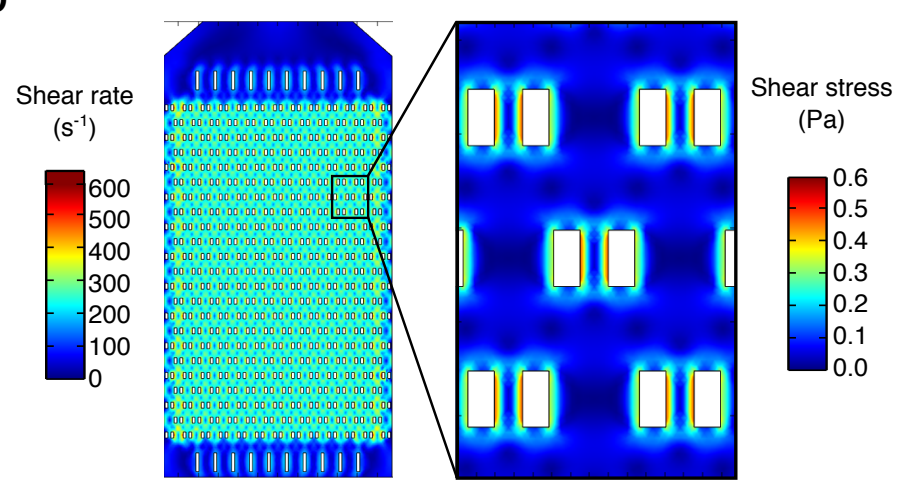

B

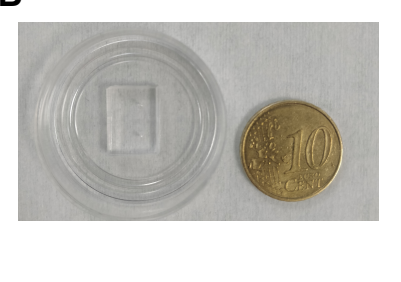

C

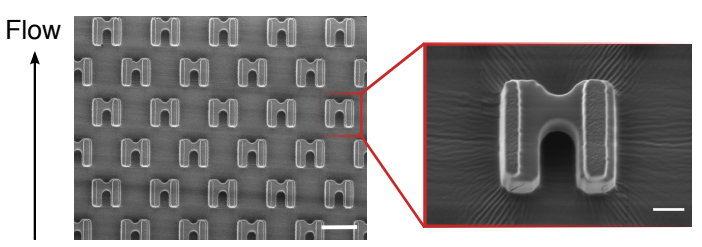

E

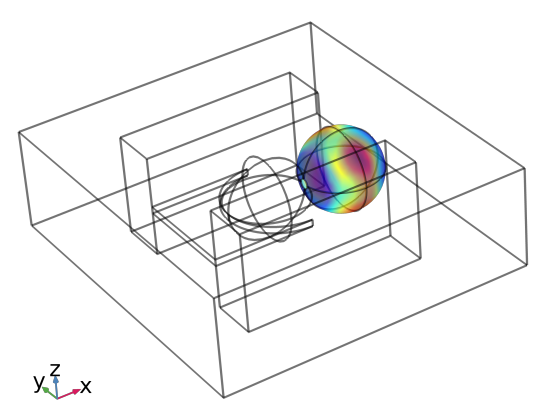

Shear stress

$(\mathrm{Pa})$

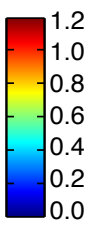

Fig. 3. Microfluidics pairing system. (A) Schematic view of the experimental setup. The microfluidic trap-based chip is imaged with an epifluorescence video-microscope, and is connected to a pressure controller inducing a low flow for droplets (then cells) circulation. (B) The PDMS chamber is sealed with a $3.5 \mathrm{~cm}$ glass-bottom Petri dish. (C) Representative SEM images of the microfluidic chamber containing 288 two-layered traps. Scale bar: $30 \mu \mathrm{m}$ (top) and $10 \mu \mathrm{m}$ (bottom). (D) $2 \mathrm{D}$ FEM simulation of fluid shear rate (left) and shear stress (right) passing through the $7 \mu \mathrm{m}$-large pillars along the whole chip, for an inlet pressure of 1000 Pa corresponding to a maximal fluid velocity of 1.6 $\mathrm{mm} / \mathrm{s}$. The shear rate is constant along the chamber. The maximal wall shear rate is about $560 \mathrm{~s}^{-1}$ that corresponds to a maximal wall shear stress about $0.56 \mathrm{~Pa}$. (E) $3 \mathrm{D}$ simulation of the shear stress that a trapped cell experiences in the microfluidic device when it is immobilized with a droplet (wireframe representation). For a maximal inlet pressure of $1000 \mathrm{~Pa}$ corresponding to a maximal fluid velocity of $1.6 \mathrm{~mm} / \mathrm{s}$ in the chamber, the maximal shear stress is about $1.0 \mathrm{~Pa}$.

(42), we consider the 10th percentile as a reasonable threshold. This results in classifying the cells with $\mathrm{I}_{\text {pol }} \geq 1.15$ as polarized. Symmetrically, we defined cells with $\mathrm{I}_{p o l}<0.85$ as anti-polarized. Consequently, non-polarized cells have $0.85 \leq \mathrm{I}_{\text {pol }}<1.15$.

B cells have different responses after being activated. Cells stimulated by droplets with standard conditions (to which we refer to as stiffness ${ }^{h i g h} /$ func $^{\text {surface }} /$ antigen $^{\text {high }}$, as detailed below), corresponding to $\gamma=12 \mathrm{mN} / \mathrm{m}$, surface functionalized, 100 antigens $/ \mu \mathrm{m}^{2}$ ) mostly polarize (55\%) forty minutes after antigen recognition (Fig. 4C, D), with only a small fraction not polarizing (15\%) (Fig. 4C and D). Surprisingly, we also noticed anti-polarization events (30\%) where lysosomes move to the opposite direction of the synapse area (Fig. 4C and D). To check that the polarization occurs specifically upon BCR engagement, we performed two major controls and compared to standard conditions: (i) we excluded an impact of the flow by checking that no cell polarized in absence of interaction at maximal flow of $1.6 \mathrm{~mm} / \mathrm{s}$ (Control \#1, S6); (ii) we excluded an activation from potential soluble antigens coming from the droplet functionalization by observing no polarized cells among the non-interacting ones in the presence of antigens-coated droplet in nearby traps (Control \#2, S6). Altogether, these results show that B cells polarize when they form a specific contact with antigen coated-soybean oil droplets suggesting that polarization is triggered by specific BCR engagement in our system.

B cell polarization depends on antigen concentration and droplet stiffness. We investigated the impact of antigen clustering, antigen concentration and droplet stiffness on lysosome polarization. We compared the cellular responses upon interaction with five different conditions of droplets: stiffness ${ }^{l o w}(\gamma=1.7 \mathrm{mN} / \mathrm{m})$, stiffness ${ }^{\text {medium }}(\gamma=4.7 \mathrm{mN} / \mathrm{m})$ or stiffness ${ }^{\text {high }}$ 
$(\gamma=12 \mathrm{mN} / \mathrm{m})$, covered with $50\left(\mathrm{Ag}^{\text {low }}\right)$ or $100\left(\mathrm{Ag}^{\text {high }}\right)$ antigen $/ \mu \mathrm{m}^{2}$, with bulk- (func $\left.{ }^{b u l k}\right)$ or surface-functionalized (func ${ }^{\text {surface }}$ ) droplets (Fig. 4C and 4D).

We first studied the mechanosensitivity of the polarization process by changing the surface tension of the droplets, hence their effective stiffness. Almost no cell (12\%, as in negative control with BSA-coated droplets) polarize with soft droplets, while more than half the cells (respectively $62 \%$ and $55 \%$ in Fig. 4D) polarize in contact with stiffness ${ }^{\text {medium }}$ and stiffness ${ }^{\text {high }}$ droplets. Above $11.7 \mathrm{kPa}$, the percentage of polarized $\mathrm{B}$ cells does not change. This result confirms that polarization is a mechanosensitive process and that the threshold of effective stiffness triggering the polarization is between 4 and $12 \mathrm{kPa}$.

We noticed that the two functionalization methods induced different antigen accumulation at the synapse: bulkfunctionalized (func ${ }^{b u l k}$ ) droplets allow clusters of antigens at the center of the synapse (Fig 4E) (similarly to what shown on other cell types (22)), while surface-functionalized (func ${ }^{\text {surface}}$ ) do not allow antigen accumulation (Fig. $\mathbf{4 F}$ and $\mathbf{4 G}$ ). Given this major difference, due to the antigen mobility (see Supplementary Note 7 for details), we characterized the effect of the type of functionalization on B cell polarization. We indeed observed the same antigen accumulation for $B$ cells as other immune cells (Fig 4E), and further quantified the kinetics of antigen accumulation with an index $I_{A g}(\mathbf{F i g} . \mathbf{4 F}) . \mathrm{I}_{A g}$ increases for antigen-coated droplets as a monotonic exponential saturation curve over $40 \mathrm{~min}$, with a characteristic time of $5 \mathrm{~min}$. The plateau is directly related to the accumulated antigen concentration: the cluster is 1.5 -fold brighter than the initial time point (Fig. 4G). We conclude that B cells are able to gather up to 75 antigens $/ \mu \mathrm{m}^{2}$ on droplets functionalized with initially 50 antigens $/ \mu \mathrm{m}^{2}\left(\mathrm{Ag}^{\text {low }}\right)$. This antigen clustering is BCR specific since no such accumulation is observed using bulk-functionalized BSA-coated droplets as negative control (Fig. 4G). Surprisingly, despite not showing antigen accumulation (Fig. 4G), func ${ }^{\text {surface }}$ droplets stimulate rather well B cell polarization regardless antigen concentration (Fig. 4C and Fig. 4D). Remarkably, func ${ }^{b u l k} / \mathrm{Ag}^{\text {low }}$ droplets, inducing antigen clustering, are able to generate the same amount of polarized cells $(52 \%)$ as func ${ }^{\text {surface }} / \mathrm{Ag}^{\text {high }}$ conditions $(55 \%)$ which initially bear twice as much antigens (respectively 50 and 100 antigens $\left./ \mu \mathrm{m}^{2}\right)$. In addition, on func ${ }^{\text {sur face }}$ droplets, when antigen concentration is lowered - from $100\left(\mathrm{Ag}^{\text {high }}\right)$ to 50 antigens $/ \mu \mathrm{m}^{2}\left(\mathrm{Ag}^{\text {low }}\right)$ - the percentage of polarized cells decreases from $55 \%$ to $35 \%$ (Fig. 4D). Altogether, these results show the dependency of B cell polarization onset on the local concentration of antigens at the synapse. The ability to aggregate ligands overcomes the lack of initial presented antigens, hence, antigen clustering lowers the threshold to established polarity when the density of presented antigens is below 75 antigens $/ \mu \mathrm{m}^{2}$.

Of note, the percentage of anti-polarized cells does not change upon presentation on substrates of different stiffness, but it does change when the antigen concentration or the ability of clustering the antigen are reduced (Fig. 4D). This suggests that the onset of anti-polarization does not depend on mechanical properties but likely on the number of engaged BCRs.

The polarization kinetic is affected by droplet mechanical properties. We assessed the impact of droplet properties on the $\mathrm{B}$ cell polarization dynamics by measuring the evolution of the polarization index $\mathrm{I}_{\text {pol }}$ over time (Fig. 5A and 5B). 
bioRxiv preprint doi: https://doi.org/10.1101/2021.12.22.473360; this version posted December 29, 2021. The copyright holder for this preprint (which was not certified by peer review) is the author/funder. All rights reserved. No reuse allowed without permission.

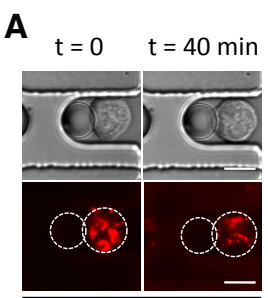

No Polarizaton

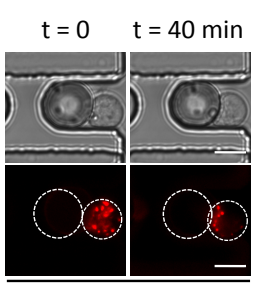

Polarizaton

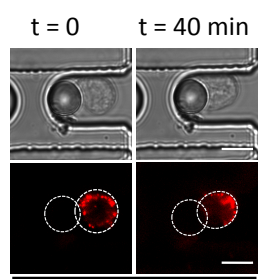

Anti-polarizaton
B

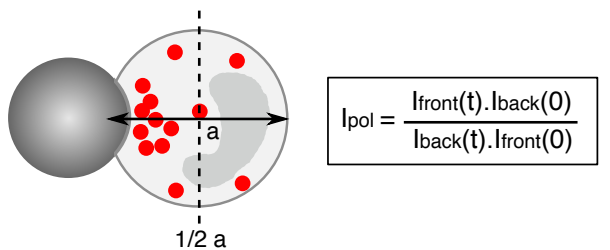

C

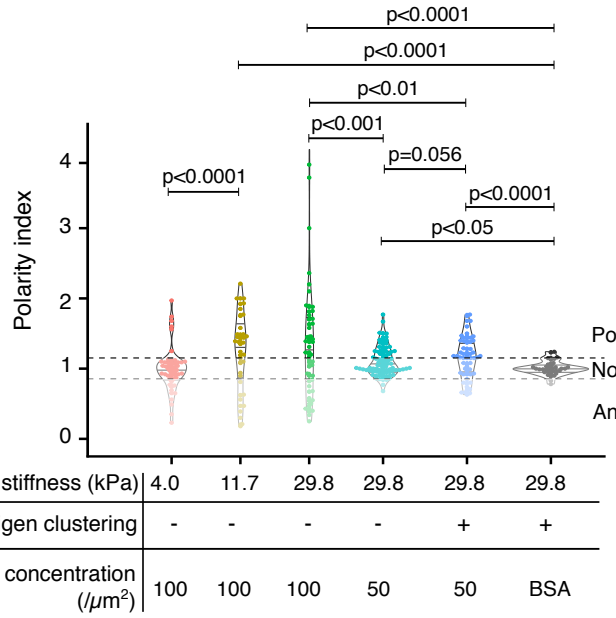

E

Bulk-functionalized $\mathrm{F}\left(\mathrm{ab} \mathrm{b}_{2}\right)_{2}$-coated droplets

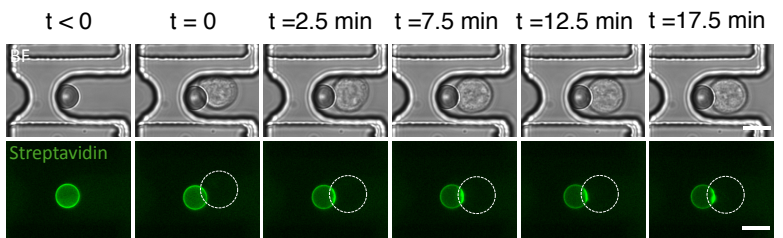

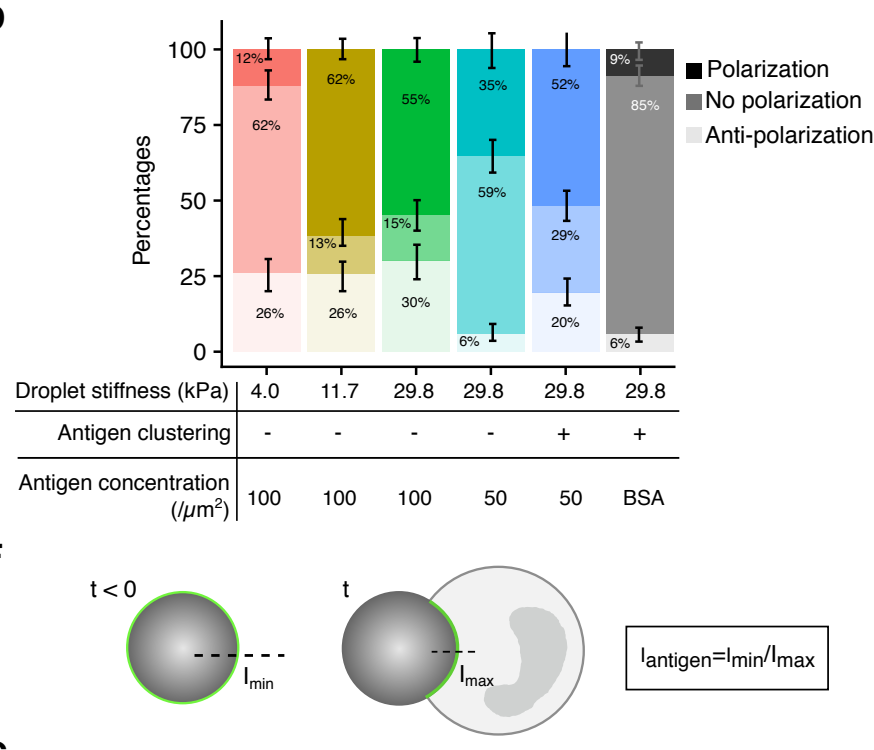

G

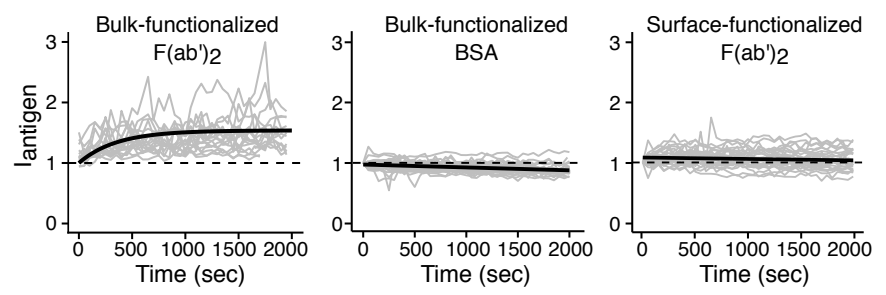

Fig. 4. B cell respond differently to droplets of different properties. (A) Representative images of the three cell behaviors 40 min after droplet encounter: non-polarized (left), polarized (center), and anti-polarized (right) lysosomes. Scalebar: $10 \mu \mathrm{m}$. (B) Schematic representation of the polarization analysis and quantification: the polarization index $\left(\mathrm{I}_{p o l}\right)$ is the ratio between the fluorescence intensity integrated over the front part, in contact with the droplet, and the fluorescence intensity integrated over the back of the cell. The ratio is normalized by its value at time zero. (C) Distribution of the $I_{p o l}, p$-values are computed by pairwise Kolmogorov-Smirnov test - when not written, the pair is not significantly different. (D) Percentages of the three cell behaviors depending on the droplet types. Error bars in graph 4D are computed as standard deviation of the percentage obtained by random subsampling 1000 groups of 15 values (typical size of the experimental pool in one day). (C)-(D) Stiffness ${ }^{l o w}$, Stiffness ${ }^{\text {medium }}$ and Stiffness ${ }^{h i g h}$ denotes quantitative stiffness of respectively 4.0,11.7 and $29.8 \mathrm{kPa}$. Antigen clustering ${ }^{+}$and Antigen clustering ${ }^{-}$denotes respectively funct $^{b u l k}$ and $^{-}$ funct $^{\text {surface }}$. Antigen concentration relative to 100 and 50 antigens $/ \mu \mathrm{m}^{2}$ denotes respectively Antigen ${ }^{\text {high }}$ and Antigen ${ }^{l o w}$. Respective number of analyzed cells from left (pink plot) to right (gray plot): $\mathrm{N}=50, \mathrm{~N}=39, \mathrm{~N}=53, \mathrm{~N}=85, \mathrm{~N}=56, \mathrm{~N}=34$, from at least 3 independent experiments) (E) Time lapse images of the antigen aggregation in a bulk-functionalized droplet - cell contact. Droplets are functionalized with $50 \mathrm{~F}\left(\mathrm{ab}^{\prime}\right)_{2}$ fragments $/ \mu \mathrm{m}^{2}$. Scalebar: $10 \mu \mathrm{m}$. (F) Scheme of antigen clustering analysis. The antigen recruitment index $\left(\mathrm{I}_{A g}\right)$ is defined as the ratio of the intensity at the time zero $\left(I_{\min }\right)$ and the intensity over time $\left(I_{\max }\right)$, both at the synapse area. $(\mathrm{G}) \mathrm{I}_{\text {antigen }}$ time evolution for three different conditions. Only bulk-functionalized antigen-coated droplets show antigen recruitment (left, $\mathrm{N}=18$ cells), while neither bulk-functionalised BSA-coated (center, $\mathrm{N}=27$ cells) nor surface-functionalized antigen-coated droplets (right, $\mathrm{N}=34$ cells) show antigen accumulation. For the bulk-functionalized antigen-coated the kinetic of antigen accumulation follows $y=(p-1)(1-\exp (-t / \tau))+1$. The related plateau $p=1.5$ allow to calculate the number of antigens aggregated at the synapse by the cell: droplets are initially coated with 50 antigens per $\mu \mathrm{m}^{2}$ and cells cluster up to $1.5 \times 50=75$ antigens $/ \mu \mathrm{m}^{2}$. 
A

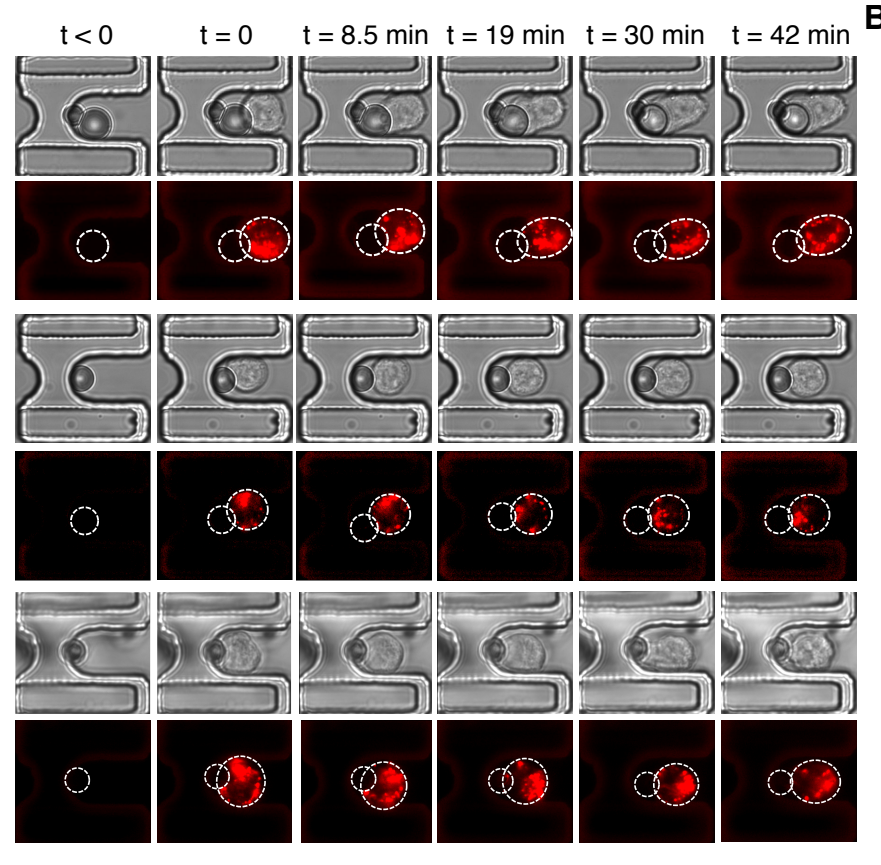

B
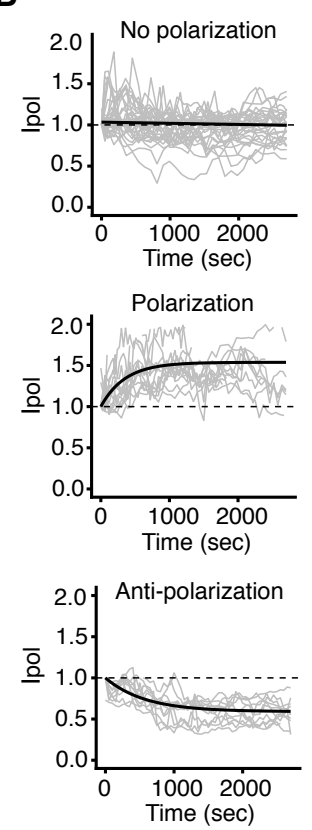

C
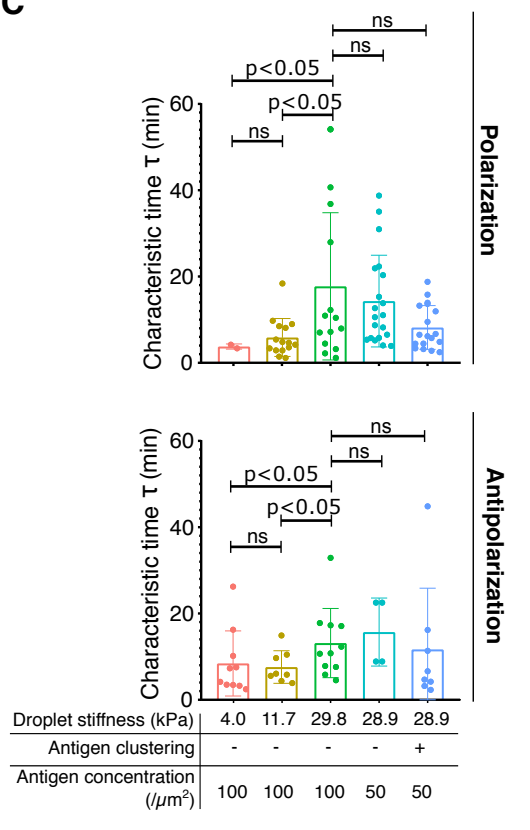

Fig. 5. B cell polarization kinetics depends on the properties of the droplets. (A) Time lapses representative of the three cell behaviors 40 min after droplet encounter: non-polarized (top, $\mathrm{N}=33$ cells), polarized (middle, $\mathrm{N}=16$ ), and anti-polarized (bottom, $\mathrm{N}=11$ ) lysosomes (from at least 3 independent experiments). (B) Fitting curves of $I_{\text {pol }}$ for polarized cells and anti-polarized cells respectively follow $y_{p o l}=(p-1)\left(1-\exp (-t / \tau)+1\right.$ and $y_{\text {antipol }}=(1-p)(1-\exp (-t / \tau))+1$ (with $\tau$ characteristic times of polarization/anti-polarization kinetics). (C) Characteristic times of polarization and anti-polarization kinetics (Mann-Whitney tests). Stiffness ${ }^{l o w}$, Stiffness ${ }^{m e d i u m}$ and Stiffness ${ }^{\text {high }}$ denotes quantitative stiffness of respectively $4.0,11.7$ and $29.8 \mathrm{kPa}$. Antigen clustering ${ }^{+}$and Antigen clustering $^{-}$denotes respectively funct $^{\text {bulk }}$ and $^{-1}$ funct $^{\text {surface. }}$.

Most of the polarizing (and anti-polarizing) kinetics follow a plateauing exponential curve, from which we can extract characteristic times $\tau$ (Fig. 5C). Figure $\mathbf{5 C}$ shows that, independently of the antigen concentrations (func ${ }^{\text {surface }} / \mathrm{Ag}^{\text {low }}$ vs. func $\left.{ }^{\text {surface }} / \mathrm{Ag}^{\text {high }}\right)$ and antigen mobility $\left(\mathrm{Ag}^{\text {low } / \text { func }} \mathrm{culk}^{\text {vs. }} \mathrm{Ag}^{\text {low } / \text { func }}{ }^{\text {surface }}\right)$, once the polarization is triggered, it reaches a plateau within $30 \mathrm{~min}$ (mean value). This suggests that polarization kinetics do not depend on the initial antigen concentration nor ability of antigen clustering as long as these conditions are sufficient to initiate the polarization. By contrast, stiffness significantly affects the polarization dynamics. In our case, B cells polarize faster in the case of stiffness $^{\text {medium }}$ than of stiffness ${ }^{\text {high }}$.

Altogether, these results reveal that B cells are dynamically mechano-sensitive, as they are able to discriminate stiffness changes, and significantly adjust the polarization rate according to the apparent stiffness of the substrates they face.

\section{Discussion}

In this work we introduced a new material to activate B cell: lipid-coated droplets. We characterized the physical and chemical properties of functionalized droplets and studied their effect on lysosome polarization in B cells. We could prove that these objects are able to activate B cells and that the onset of polarization is sensitive to ligand concentration and rigidity. Unexpectedly we show that the kinetic of polarization is also sensitive to this last property. This is consistent with the current model of antigen internalisation. In fact lysosome polarization has been shown to be necessary for protease secretion, antigen degradation and extraction (11); it has also been shown that this mode of antigen extraction occurs only when mechanical pulling fails (13); this suggests that a stiff non-deformable substrate, from which the antigen cannot be ripped off, induces lysosome polarization more easily than a soft/deformable substrate. Thus, our work shows that the 
establishment and the kinetics of polarization both depend on the mechanical properties of the droplet.

Role of BCR mechanosensitivity. We show that B cells polarize only when they interact with substrates presenting a surface tension greater than $4.7 \mathrm{mN} / \mathrm{m}$ (corresponding to an apparent stiffness of $11.7 \mathrm{kPa}$ ). This is consistent with observation on macrophages and follicular dendritic cells (13). Of note, the stiffest (11.7 and $29.8 \mathrm{kPa})$ and softest (4.0 kPa) droplets induce responses similar to, respectively, follicular dendritic cells and dendritic cells (13). We propose that this is linked to the BCR mechanosensitivity. Indeed, BCR signal is known to depend on the substrate stiffness (5) and the signal downstream the BCR to induce the accumulation of polarity cues $(3,10,14)$. These polarity cues establish the pole by anchoring the centrosome (43), reorienting the microtubule based traffic towards the synapse: the stronger the signal, the stronger the establishment of polarity cues, the higher the probability of polarizing.

The rate of polarization is mechanosensitive. The dependency of the polarization kinetics on droplet stiffness suggests that the rate of polarization is also mechanosensitive. Mechanosensitivity of dynamical properties has been shown in adhesive cells: actin flows increase with the rigidity of the substrate (44), cell adapt their contractility in real time to the stiffness change of the substrate (45), and T cells adapt their pulling force at the synapse to the stiffness of the interacting object (46). However, the speed of polarization is likely the result of an integration of several signals and less directly related to the cytoskeletal changes. One explanation could be that the increasing in stiffness (47), elicited by BCR activation, hinders the displacement of the centrosome and hence the polarization of lysosomes, effectively lowering the polarization rate on the stiffest substrates.

Role of antigen mobility and concentration. We quantified the statistics of cell polarization when they were able to accumulate antigens at the contact zone or not, and to interact with 50 to 100 antigens $/ \mu \mathrm{m}^{2}$. Our results showed that the maximal amount of polarized cells is reached for 75 antigens $/ \mu \mathrm{m}^{2}$, suggesting that above this density, antigen concentration has no effect on the polarization process. This value is compatible with the maximal density of BCRs available in an activated synapse. In addition, previous work reported that antigen concentration from 15 to 150 antigens $/ \mu \mathrm{m}^{2}$ modulates the $B$ cell activation with a threshold that depends on the affinity (8). Although we cannot quantitatively compare these results to ours (different antigens and presentation assays), this suggests the concentration threshold for polarization might also depend on the antigen affinity. Further experiments will better elucidate the link between early signaling, lysosome polarization, and antigen affinity. Other works have shown that B cells interacting with mobile ligands displayed significantly greater signaling because of the formation, the accumulation, and the merging of BCRs microclusters $(9,48,49)$. We herein show that lysosomes polarize independently of the ability to form a central antigen cluster. We showed, however, that "mobility", allowing the local increase of antigen concentration at the synapse, helps B cells overcome the low initial antigen concentration (below 75 antigens $/ \mu \mathrm{m}^{2}$ ) and increases the chance for a cell to polarize. This suggests that local antigen concentration is a more crucial feature than mobility for inducing polarization.

Anti-synaptic lysosome polarization. One perk of our system is to allow the study of cell-to-cell variability. Even in clonal B cell lines and using highly monodisperse and homogeneous coated droplets, we show that not all cells polarize their 
lysosome towards the synapse, but some even displace them in the opposite side of the synapse (anti-polarize). In T lymphocytes, a transient anti-polarisation of actin and myosin has been observed in the early stage of the T cell/APC contact, and does not depend on antigen recognition (50). By contrast, B cell anti-polarization is stable (over $40 \mathrm{~min}$ ), BCR specific and is only triggered above 75 antigens $/ \mu \mathrm{m}^{2}$. Interestingly, the stiffness does not influence the onset of anti-polarization. Like the polarization process, antigen mobility and concentration does not affect the anti-polarization kinetics, but stiffness does. Of note, we observed that anti-polarized cells are more elongated and considerably less round than the polarizing or non responsive ones. Similar events have been described in frustrated T cell-APC conjugates (51), where the centrosome is blocked behind the nucleus while membranes accumulate at the synapse, which ultimately deforms the cell. Alternatively, the anti-synapse might represent a signaling complex in its own right as already described in T cells (50). Further comparison of the proteins implicated in the proximal and distal poles, both during the polarization and the anti-polarization formation, would elucidate this latter suggestion.

Perspectives. Our method allowed us to precisely phenotype lysosome polarization dynamics. This may be combined with classic (genetic or pharmacological) perturbations to dissect single cell polarization mechanisms in real time. We anticipate that this approach will be of interest in many other contexts, where cell-cell variability is important for the immune function such as T cell immune synapses $(52)$, cytotoxic cell encounter $(53,54)$, and phagocytosis $(22,55)$.

\section{Materials and methods}

\section{Materials}

Pluronic F-68 (Poloxamer 188, CAS no. 9003-11-6), sodium alginate (CAS no. 9005-38-3), Tween 20 (polyethylene glycol sorbitan monolaurate, CAS no. 9005-64-5), oleic acid (CAS no. 112-80-1, ref. 01008), silicone oil (viscosity 350 cSt at $25{ }^{\circ} \mathrm{C}$, CAS no. 63148-62-9) and mineral oil (light oil, CAS no. 8042-47-5) were purchased from Sigma-Aldrich (Saint Quentin Fallavier, France). DSPE-PEG 2000 -Biotin lipids (1,2 - distearoyl - sn - glycero - 3 - phosphoethanolamine - N - [biotinyl (polyethyleneglycol) - 2000] ammonium salt, CAS no. 385437-57-0) were purchased from Avanti Polar Lipids (Alabaster, AL, U.S.A). FluoProbes 488 streptavidin (ref. FP-BA2221) and biotin goat anti-mouse IgG F(ab') fragment $^{2}$ MIN x (HU, BOV, HRS) were respectively purchased from Interchim (Montlucon, France) and Jackson ImmunoResearch (Ely, U.K.). Ultrapure water (Millipore, 18.2 M.cm) was used for all experiments. All reagents and materials were used as purchased, without any further purification. Lipiodol (CAS no. 8002-46-8) was kindly provided by the company Guerbet (Villepinte, France).

\section{Cell culture}

Mouse IgG+ B-lymphoma cell lines IIA1.6 (26), derived from A20 cell lines (ATTC\# TIB-208), were cultured at $37^{\circ} \mathrm{C}$ in a 5\% CO2 atmosphere in CLICK medium (RPMI 1640 Medium GlutaMAXTM Supplement, ref. 61870036; supplemented with $10 \% \mathrm{v} / \mathrm{v}$ decomplemented fetal calf serum, ref. 16140063; 1\%v/v antibiotic Penicillin-Streptomycin, ref. 15070063; $2 \% \mathrm{v} / \mathrm{v}$ sodium pyruvate at $100 \mathrm{mM}$, ref. 11360070 ; and $0.1 \% \mathrm{v} / \mathrm{v} 2-$ Mercaptoethanol at $50 \mathrm{mM}$, ref. 31350010). All these 
reagents were purchased from Life Technologies - Gibco. Experiments were performed with cell densities close to 106 cells $/ \mathrm{mL}$, which were diluted to ca. 200000 cells $/ \mathrm{mL}$ and split in 10 samples of $1 \mathrm{~mL}$, inserted in a 12-well plate (Falcon, ref. 353043). For each experiment, a $1 \mathrm{~mL}$ cell sample and $25 \mu \mathrm{L}$ of HEPES (4- (2-hydroxyethyl) - 1- piperazineethanesulfonic acid, Gibco) were put in a $1.8 \mathrm{~mL}$ tube compatible with the pressure controller (Fluigent, France). In the meantime, other samples remained incubated at $37^{\circ}, 5 \%$ of $\mathrm{CO} 2$ for a maximum of $4 \mathrm{~h}$.

\section{Cell staining}

Lysosomes were stained by loading the cells with LysoTrackerTM Red DND-99 (1 mM, ref. L7528) at a final concentration of $50 \mathrm{nM}$ purchased from Life Technologies | Thermo Fisher Scientific (Invitrogen).

\section{Bulk droplet functionalization protocol with phospholipids}

The lipid-containing oil was obtained by dilution of DSPE-PEG 2000 -Biotin phospholipids (Avanti Lipids, Alabama, USA) in soybean oil at $0.03 \mathrm{mg} \cdot \mathrm{mL}^{-1}$, followed by $30 \mathrm{~min}$ of sonication and evaporation of the chloroform from the oil at room temperature. This oil was dispersed and emulsified by hand in an aqueous continuous phase containing $15 \% \mathrm{w} / \mathrm{w}$ of Pluronic F68 block polymer surfactant and 1\% w/w sodium alginate (Ref : W201502, Sigma Aldrich, St. Louis, MO, USA) at a final oil fraction equal to $75 \% \mathrm{w} / \mathrm{w}$. The rough emulsion was then sheared in a Couette cell apparatus at a controlled shear rate following the method developed by Mason et al. (28) to narrow the droplet size distribution to $12.4 \mu \mathrm{m} \pm 2.3$ $\mu \mathrm{m}$. For storage, the emulsions are diluted to an oil fraction of $60 \mathrm{wt} \%$ with $1 \mathrm{wt} \%$ Pluronic F68 in the continuous phase and stored at $12^{\circ} \mathrm{C}$ in a Peltier storage cupboard for several weeks.

\section{Surface Droplet Functionalization Protocol with Phospholipids}

Droplets were formulated using a Shirasu Porous Glass apparatus (SPG Technology Co., Japan) by extruding the oil phase (with or without $5 \% \mathrm{v} / \mathrm{v}$ of oleic acid) through a ceramic membrane (with pores of $3.1 \mu \mathrm{m}$, SPG Technology Co., Ltd) within an aqueous solution containing $15 \% \mathrm{v} / \mathrm{v}$ of Pluronic F68, continuously and gently stirred. We obtained droplets measuring $10.98 \pm 0.68 \mu \mathrm{m}$ in diameter. Once stabilized, droplets were washed out 3 times with an aqueous solution of Tween 20 at CMC $(0.0007 \% \mathrm{w} / \mathrm{v})$. The supernatant was removed and replenished after each centrifugation step (30 sec at 2000 RPM). The suspension is centrifuged and rinsed four times with phosphate buffer (PB) to decrease the amount of Pluronic F68 in the continuous phase. After the last rinsing step, most of the continuous phase is removed from the microtube to decrease the total volume of emulsion to $10 \mu \mathrm{L}$. We then add $10 \mathrm{vol} \%$ of the DMSO stock solvent solution containing the phospholipids. We then add a phosphate buffer supplemented with Tween 20 at the CMC (PB - Tw20) to reach a total volume of $200 \mu \mathrm{L}$ of suspension. Droplets are incubated for $30 \mathrm{~min}$ at room temperature in the presence of lipids in the bulk phase and rinsed several times with a PB - Tween 20 buffer to remove the phospholipids in excess. The quantity of phospholipids available in the bulk phase at the initial stage is adjusted by diluting the stock solutions in DMSO so that the volume fraction of cosolvent remains constant for all experimental conditions. For a given working volume (200 $\mu \mathrm{L}$ ), the lipid concentration in the bulk phase is expressed as a droplet surface area equivalent. One equivalent (eq.) of molecules corresponds to the number of molecules necessary to cover the total area of a droplet sample with a compact 
monolayer of molecules (Pinon, 2018).

\section{Coating of Biotinylated Droplets with Streptavidin and $F\left(a^{\prime}\right)_{2}$ fragments}

After the first step of emulsification and insertion of biotinylated lipids at the interface, 1 equivalent of streptavidin solution at $1 \mathrm{mg} / \mathrm{mL}$ is added, followed by an incubation time of $30 \mathrm{~min}$ and a washing step to remove all excess streptavidin. Finally, 1 equivalent of $F\left(a b^{\prime}\right)_{2}$ fragments $(1 \mathrm{mg} / \mathrm{mL})$ or $B S A(1 \mathrm{mg} / \mathrm{mL})$ is added to the droplet solution, followed by an incubation time of $30 \mathrm{~min}$ and a washing step to remove all excess $\mathrm{F}\left(\mathrm{ab} \mathrm{b}^{\prime}\right)_{2}$ or BSA. One equivalent (1 eq.) of a given macromolecule (lipid or protein) corresponds to the theoretical number of molecules needed to cover the entire surface with a monolayer of molecules or proteins, considering the available surface of droplet and molecule dimensions as previously reported in (30).

\section{Measurement of the adsorption isotherms of the surface-functionalized droplets}

To quantify the amount of antigens presented by droplets, we first titrated the antigens (from 0.05 to 5 eq.) bound to streptavidin molecules (1 eq.) and biotinylated DSPE-PEG 2000 lipids (100 eq.). We revealed the antigens coating with a Goat anti-Rabbit IgG (H+L) Cross-Adsorbed Secondary Antibody (Alexa Fluor 647, Invitrogen, ref. A-21244) and analyzed the fluorescence intensity. In our case, the droplets are saturated for 4 eq. of $F\left(a b{ }^{\prime}\right)_{2}$ fragments (Fig. 1E). We then converted this relative concentration into absolute value by comparing fluorescence intensity of droplets and commercial tagged beads (Quantum TM MESF, Bang Laboratories, Inc.). We assessed the related fluorescence intensities of droplets coated with a concentration of streptavidin ranging from 0 to 2 eq. and saturated in biotinylated DSPE-PEG 2000 lipids (100 eq.) (Fig 1D). These values are added on a calibration curve correlating the molecular equivalent surface fluorescence (MESF) of beads (Quantum ${ }^{\mathrm{TM}}$ MESF, Bang Laboratories, Inc.) with their fluorescence intensities (Fig. S1). Finally, we converted the droplet MESF into absolute values of $\mathrm{F}\left(\mathrm{ab}^{\prime}\right)_{2}$ fragments in antigens per $\mu \mathrm{m}^{2}$ (Fig. 1F) depending on different droplet and protein features. We obtained that droplets present from 0 to 150 antigens $/ \mu \mathrm{m}^{2}$ when they are functionalized with DSPE-PEG 2000 -Biotin (100 eq.), streptavidin from 0 to 2 eq. and $F\left(a b{ }^{\prime}\right)_{2}$ fragments (4 eq.). For the rest of the experiment, we uses droplets coated with 50 and 100 antigens $/ \mu \mathrm{m}^{2}$.

\section{Measurement of oil/water interfacial tension by the pending drop technique}

We used a pending drop apparatus (drop shape analyzer, Krüss, PSA30) to measure the interfacial tension of the various oils considered in this study - mineral oil, mineral oil supplemented with $5 \% \mathrm{v} / \mathrm{v}$ of oleic acid, soybean oil, lipiodol and lipiodol supplemented with $5 \% \mathrm{v} / \mathrm{v}$ of oleic acid - versus an aqueous solution composed of $15 \% \mathrm{w} / \mathrm{w}$ Pluronic 68 . The interfacial tensions reported in Table 1 correspond to the equilibrium value reached when the drop surface has been entirely covered by surfactants coming from the continuous phase.

\section{Measurement of droplets interfacial tension by the micropipette technique}

The micropipette-aspiration method that has been described in detail in a previous article (56). Micropipettes were made from $1 \mathrm{~mm}$ borosilicate glass-tube capillaries (Harvard Apparatus, USA) that were pulled in a pipette heater and puller 
(P-2000, Sutter instrument Co., USA) to tip diameters in the range of 3 to $5 \mu \mathrm{m}$. A 3 axis-micromanipulator (Narishige) allowed for pipette positioning and manipulation. The pipette was connected to a pressure controller (Fluigent) to apply precise negative pressures. A solution of sinking droplets, made with lipiodol oil coated or not with phospholipids, was inserted into a glass/coverslip chamber. The pipette aspired the droplet until reaching an equilibrium where the elongation part is equal to the pipette inner dimension. The interfacial tension $\gamma$ is $\gamma=\left(\Delta P \cdot\left(R_{p} / 2\right)\right.$ where $\Delta P$ is the negative pressure and $\mathrm{R}_{p}$ the micropipette radius. Upon aspiration by the very thin glass pipette, the droplet deforms and a spherical cap of radius $R_{c}$ forms at the tip of the pipette. At equilibrium, the value of $R_{c}$ depends on the interfacial tension $\gamma$ of the droplets, the radius of the droplet $R_{D}$, the aspiration pressure $\Delta \mathrm{P}$ and can be expressed as:

$$
\frac{1}{\mathrm{R}_{C}}=\frac{\Delta \mathrm{P}}{2 \gamma}+\frac{1}{\mathrm{R}_{D}}
$$

The aspiration $\Delta \mathrm{P}$ corresponds to the pressure difference between the inside of the pipette and the external pressure. $\gamma$ and $R_{p}$ are constant throughout the experiment. Hence, varying $\Delta \mathrm{P}$ induces changes in $\mathrm{R}_{c}$ : the larger $\Delta \mathrm{P}$, the smaller $R_{c}$. During measurements, $\Delta \mathrm{P}$ is slowly increased and $\mathrm{R}_{c}$ decreases until reaching the radius of the pipette $\mathrm{R}_{p}$. Up to that critical aspiration $\Delta \mathrm{P}_{c}$, little change is observed in the geometry of the system. As soon as $\Delta \mathrm{P}$ becomes greater than $\Delta \mathrm{P}_{c}, \mathrm{R}_{c}$ becomes smaller than $\mathrm{R}_{p}$, which results in the sudden entry of the oil droplet in the pipette. This provides a direct measurement of the surface tension of the droplet:

$$
\gamma=\frac{\Delta \mathrm{P}_{c}}{2\left(\frac{1}{\mathrm{R}_{D}}-\frac{1}{\mathrm{R}_{p}}\right)}
$$

\section{Measurement of droplet dynamic interfacial tension by the microplate technique}

Microplates were made from glass lamellae of $100 \times 2 \mathrm{~mm}$ with $0.1-0.3 \mathrm{~mm}$ thickness. The rigid plate can be either pulled out of 0.2 or $0.3 \mathrm{~mm}$ original thickness, but must be kept short to ensure a high stiffness. Lamellae were heated and pulled (Narishige PB-7, Japan) until breaking in two similar parts. Microplates were calibrated using a microplate of known bending stiffness as a reference. The bending stiffness of the reference microplate has been initially determined by stacking copper micro-wires at the extremity of the plate, following the protocol reported in (57). A microplate of low bending stiffness (flexible) acts as a spring, and a rigid plate about 1000 times stiffer than the flexible one acts as support. During experiments, the flexible plate is oscillated, applying oscillating stress on the studied sample and compressing it sinusoidally against the rigid plate. The flexible plate was oscillated from 0.1 to $6.4 \mathrm{~Hz}$ for droplets and until $1.6 \mathrm{~Hz}$ for cells. Micrometric displacements of plates are controlled via highly-resolutive piezoelectric micromanipulators. The deflection $\delta$ of the flexible plate is related to the force $F$ exerted on the sample by $F=k \delta$ where $k$ is the bending stiffness of the flexible plate. The deformation of the sample is $\epsilon=\left(L_{0}-L\right) / L_{0}$ where $L_{0}$ is the resting cell or droplet diameter (Fig. 2A). For mechanical characterization, we used the dynamic analysis protocol consisting in applying a controlled sinusoidal stress or strain at different frequencies on the cell, while measuring the complementary visco-elastic response provided by the droplet or cell (57). For droplet experiments, Lipiodol (Guerbet) oil was used to fabricate droplets and glass microplates 
were coated with PLL at $0.1 \% v / v$. For cells, only the chamber was coated with Sigmacote (Sigma-Aldrich).

\section{Design and microfabrication of the microfluidic trapping array}

Microfluidic chips were designed on CleWin (WieWeb Software). The double layer technique required the creation of two masks: traps and pillars. Chrome masks were fabricated with the $\mu \mathrm{PG} 101$ maskless aligner (Heidelberg Instruments Mikrotechnik $\mathrm{GmbH}$ ), and the final silicon mold with the MJB4 aligner system (Karl Süss). Two SU8 photoresists (Microchem) have been used, 2005 and 2010 to respectively obtain $5 \mu$ m-thick pillars and $10 \mu \mathrm{m}$-thick traps. To avoid any PDMS sticking to the small SU8 structures and long-term mold damage, we coated the wafer with a fluorinated silane by vapor deposition (trichloro (1H,1H,2H,2H-perfluorooctyl) silane, CAS: 102488-49-3, Merck-Sigma-Aldrich). Liquid PDMS (RTV 615, Momentive Performance) at a base:crosslinker 1:10 ratio was poured on the silicon-SU8 mold and cured for more than $2 \mathrm{~h}$ at $70^{\circ} \mathrm{C}$ to complete the crosslinking. After cutting PDMS pieces and punching out inlets and outlets with a biopsy puncher (OD $=0.75 \mathrm{~mm}$, Electron Microscopy Sciences, Hatfield, UK), the top PDMS part was bonded to a glass-bottom Petri dish (FluoroDish FD35-100, WPI) together after a $\mathrm{O}_{2}$ plasma treatment of both surfaces $(50 \mathrm{~W}$ for $30 \mathrm{~s}$, $20 \mathrm{sccm} \mathrm{O}_{2}$ flow, 0.15 torr pressure, Cute Plasma oven, Femto Science, Korea), and left for $30 \mathrm{sec}$ at $90^{\circ} \mathrm{C}$ to improve the bonding. For a long-term hydrophilic coating (Hemmila, 2012) of the inner PDMS channels, a 0.25 wt\% polyvinylpyrrolidone (PVP, K90, Sigma Aldrich) solution was injected in the chip. Finally, the chip channels were rinsed with culture media before inserting droplets and cells.

Experimental setup for the cell-droplet encounters. By connecting the microfluidic devices to a pressure controller, a fixed pressure drop $\Delta \mathrm{P}$ between the inlet and the outlet of the chamber is set (maximum $1000 \mathrm{~Pa}$, corresponding to a fluid velocity of $1.6 \mathrm{~mm} / \mathrm{s})$. Traps are initially rinsed with CLICK medium during at least $5 \mathrm{~min}$. Then, $200 \mu \mathrm{L}$ of the droplets suspension $\left(10^{6}\right.$ droplets $/ \mathrm{mL}$ in CLICK medium) are inserted in the microchip using the pressure regulator. Once a desired number of droplets is trapped, the first tubing is carefully removed and replaced by the tubing connected to the $B$ cell-containing tube. Different positions and focus are marked and cells are progressively inserted at a maximum speed of $1.6 \mathrm{~mm} / \mathrm{s}$ while the acquisition is launched. To avoid a saturation of traps in cells while ensuring culture medium replenishment, the flow is lessened at $0.4 \mathrm{~mm} / \mathrm{s}$. Particle displacements and trapping are observed by video-microscopy.

\section{Microscopy-Imaging of B cell polarization dynamics}

Brightfield and fluorescent images of the synapses are acquired on a Leica DMI8 microscope (Germany) connected to an Orca Flash4.0 sCMOS camera (Hamamatsu Photonics, Japan). Epi-illumination is done with a LED light (PE-4000, CoolLED) and a GFP filter set (Excitation/Emission: 470/525 nm) for the fluorescent coated droplets, and a TexasRed filter set (Excitation/Emission : 561/594 nm) for the fluorescent cells. Time zero of the experiment is defined manually when a cell encounters a droplet. All pictures of cell-droplet pairs were imaged with a 40x objective (Leica, dry, N.A.=0.8). 


\section{Image Analysis}

Image analyses were performed with ImageJ/ Fiji (58) (version 1.52i), and data analyses were performed with R (RStudio) software (version 2). For all processes, 16-bit images were analyzed. Codes are available on request.

\section{Quantification of antigen recruitment}

The recruitment index is defined as the ratio between the fluorescence intensity over time I $(t)$ divided by the fluorescence intensity at the initial time I $(0)$, both at the synapse area. The synapse area is countered by hand for each time point. The maximum and mean fluorescence intensities were measured at each time point (from time zero to $40 \mathrm{~min}$ ).

\section{Quantification of lysosome polarization}

Each cell shape was contoured by hand for each time point (from time zero to $40 \mathrm{~min}$ ) and using a ImageJ macro, automatically divided in two parts according to the main orientation of the cell-droplet pair. It extracted the fluorescence intensity at the middle front side of the cell (in contact with droplet) $\mathrm{I}_{\text {front }}(t)$ and at the back of the cell $\mathrm{I}_{\text {back }}(t)$ at each time point, and computed the polarization index defined as $\left[\mathrm{I}_{\text {front }}(t) / \mathrm{I}_{\text {back }}(t)\right] \cdot\left[\mathrm{I}_{\text {back }}(0) / \mathrm{I}_{\text {front }}(0)\right]$ (Fig. 4D).

\section{Numerical simulations}

The numerical simulations are performed with COMSOL Multiphysics $®($ version 5.3). We first developed a twodimensional depth-averaged computational fluid dynamics - finite element model of the microfluidic chamber with exactly the same approach that the one proposed in (59). We consider a planar and incompressible flow, at steady state, and a Newtonian fluid with a dynamic viscosity of $\mu=10^{-3}$ Pa.s. Hence, the equation set verified inside the fluid domain is written as $-\nabla \mathrm{P}+\mu \Delta v+f_{v}=0$ and $\nabla \cdot v=0$, where $\mathbf{P}$ is the pressure field, $v$ the velocity field and $f_{v}$ the volume force. In addition, the non-slip condition on the out-of-plane walls is considered with a Darcy's law (also called "shallow channel approximation") thus follows $f_{v}=-12 \mu / d^{2} v$.

On physical walls, a non-slip boundary condition is added. The pressure is set as uniform both at the inlet and outlet. This pressure is equal to the pressure drop in the chamber or equal to $0 \mathrm{~Pa}$, respectively at the inlet and outlet. Finally, we deduced the pressure drop through a single trap $\Delta \mathrm{P}_{\text {trap }}$ from the isobar lines plot (Fig. S4A).

A 3D model focused on the geometry of one trap. We used the same hypotheses and set of equations than the previously detailed 2D model except that we do not consider Darcy's law, thus we consider a non-slip condition on the upper and lower faces of the chamber as $f_{v}=0$. On the inlet, the pressure is set as uniform and equal to $\Delta \mathbf{P}_{\text {trap }}$ while on the outlet, it is considered as uniform and equal to $0 \mathrm{~Pa}$. Finally, we considered the flow as symmetric on the boundary linking with the rest of the microfluidic chamber.

\section{Statistical analysis}

All statistical analysis have been performed using Prism GraphPad (version 8) and R programming language. Codes are available on request. Tests and $p$-values are specified in the figures. 
bioRxiv preprint doi: https://doi.org/10.1101/2021.12.22.473360; this version posted December 29, 2021. The copyright holder for this preprint (which was not certified by peer review) is the author/funder. All rights reserved. No reuse allowed without permission.

\section{ACKNOWLEDGEMENTS}

The authors thank A.M Lennon-Duménil for scientific support and critical reading of the manuscript, thank C. Guerin and A. Chipont of Institut Curie's cytometry platform for fruitful discussions and technical help, and the company Guerbet for having kindly provided Lipiodol@oil samples, F. Pincet, D. Langevin, B. Cabane and J. Voldman for helpful discussions, P. Saez for cell culture advice and O.Ait-Mohamed for help with statistical analysis. This work benefited from the technical contribution of the Institut Pierre-Gilles de Gennes joint service unit CNRS UAR 3750. The authors would like to thank the engineers of this unit for their advice during the development of the experiments. The authors are grateful to H. Moreau, M. Thery and R. Dreyfus for critical reading of the paper and suggestions.

\section{AUTHOR CONTRIBUTIONS}

L.P., P.P. and J.F. designed the study. L.P. performed all the experiments and analyzed experimental results. N.R and R.A. conducted all simulation studies. O.M carried out preliminary experiments on microfluidics and cell culturing. J.P. helped during microfluidics and cell culturing experiments. D.C. made the micropipette setup and contributed to the experiments. S.A and A.T performed all microplate experiments, analyzed experimental results and wrote the manuscript. L.P., P.P and J.F. wrote the manuscript. All authors approved the final version of the article.

\section{FUNDINGS}

L.P., N.R. and J.P. were respectively funded by the IPV scholarship program (Sorbonne Université), the École Normale Superieure de Rennes, and the Bettencourt scholarship program (CRI-FIRE).J.F. acknowledges funding from the Agence Nationale de la Recherche (ANR Jeune Chercheur PHAGODROP, ANR-15- CE18-0014-01).

\section{COMPETING FINANCIAL INTERESTS}

No competing financial interests

\section{Bibliography}

1. Johannes B. Huppa and Mark M. Davis. T-cell-antigen recognition and the immunological synapse. Nature Reviews Immunology, 3(12):973-983, 2003. ISSN 14741733. doi: 10.1038/nri1245.

2. M.-I. Maria-isabel Yuseff, Paolo Pierobon, Anne Reversat, and A.-M. Lennon-Duménil. How B cells capture, process and present antigens : a critical role for cell polarity Antigen encounter and the role of presenting Cells. Nature Reviews Immunology, 13(July):475-486, 2013. ISSN 14741733. doi: 10.1038/nri3469.

3. Karen L. Angus and Gillian M. Griffiths. Cell polarisation and the immunological synapse. Current Opinion in Cell Biology, 25(1):85-91, 2013. doi: 10.1016/J.CEB.2012.08.013.

4. F. D. Batista and M. S. Neuberger. Affinity Dependence of the B Cell Response to Antigen: A Threshold, a Ceiling, and the Importance of Off-Rate. Cell Press, 8:751-759, 1998.

5. Z. Wan, S. Zhang, Y. Fan, K. Liu, F. Du, A. M. Davey, H. Zhang, W. Han, C. Xiong, and W. Liu. B Cell Activation Is Regulated by the Stiffness Properties of the Substrate Presenting the Antigens. The Journal of Immunology, 190(9):4661-4675, 2013. ISSN 0022-1767. doi: 10.4049/jimmunol.1202976.

6. Samina Shaheen, Zhengpeng Wan, Zongyu Li, Alicia Chau, Xinxin Li, Shaosen Zhang, Yang Liu, Junyang Yi, Yingyue Zeng, Jing Wang, Xiangjun Chen, Liling Xu, Wei Chen, Fei Wang, Yun Lu, Wenjie Zheng, Yan Shi, Xiaolin Sun, Zhanguo Li, Chunyang Xiong, and Wanli Liu. Substrate stiffness governs the initiation of B cell activation by the concerted signaling of PKC $\beta$ and focal adhesion kinase. eLife, 6:1-29, 2017. ISSN 2050084X. doi: 10.7554/eLife.23060.

7. Y. R. Carrasco, S. J. Fleire, T. Cameron, M.I L. Dustin, and F. D. Batista. LFA-1/ICAM-1 interaction lowers the threshold of B cell activation by facilitating B cell adhesion and synapse formation. Immunity, 20(5):589-599, 2004. ISSN 10747613. doi: 10.1016/S1074-7613(04)00105-0.

8. S. J. Fleire, J. P. Goldman, Y. R. Carrasco, M. Weber, D. Bray, and F. D. Batista. B cell ligand discrimination through a spreading and contraction response. Science, 312(5774):738-741, 2006. ISSN 00368075. doi: 10.1126/science.1123940.

9. Christina Ketchum, Heather Miller, Wenxia Song, and Arpita Upadhyaya. Ligand mobility regulates B cell receptor clustering and signaling activation. Biophysical Journal, 106(1):26-36, jan 2014. ISSN 1542-0086. doi: 10.1016/j.bpj.2013.10.043.

10. Anne Reversat, Maria-Isabel Yuseff, Danielle Lankar, Odile Malbec, Dorian Obino, Mathieu Maurin, Naga Venkata Gayathri Penmatcha, Alejandro Amoroso, Lucie Sengmanivong, Gregg G Gundersen, Ira Mellman, François Darchen, Claire Desnos, Paolo Pierobon, and Ana-Maria Lennon-Duménil. Polarity protein Par3 controls B-cell receptor dynamics and antigen extraction at the immune synapse. Molecular biology of the cell, 26(7):1273-85, apr 2015. ISSN 1939-4586. doi: 10.1091/mbc.E14-09-1373.

11. M. I. Yuseff, A. Reversat, D. Lankar, J. Diaz, I. Fanget, P. Pierobon, V. Randrian, N. Larochette, F. Vascotto, C. Desdouets, B. Jauffred, Y. Bellaiche, S. Gasman, F. Darchen, C. Desnos, and A.-M. Lennon-Duménil. Polarized Secretion of Lysosomes at the B Cell Synapse Couples Antigen Extraction to Processing and Presentation. Immunity, 35(3):361-374, 2011. ISSN 10747613. doi: 10.1016/j.immuni.2011.07.008.

12. E. Natkanski, Justin E. Molloy, and P. Tolar. B Cells Use Mechanical Energy to Discriminate Antigen Affinities. Science, 340(June):1587-1590, 2013.

13. K. M. Spillane and P. Tolar. B cell antigen extraction is regulated by physical properties of antigen presenting cells. Journal of Cell Biology, 2(1):1-19, 2016. ISSN 00063495. doi: 10.1016/j.bpj. 2016.11.701.

14. J. Wang, F. Lin, Z. Wan, X. Sun, Y. Lu, J. Huang, F. Wang, Y. Zeng, Y. H. Chen, Y. Shi, W. Zheng, Z. Li, C. Xiong, and W. Liu. Profiling the origin, dynamics, and function of traction force in B cell activation. Science Signaling, 11(542), 2018. ISSN 19379145. doi: 10.1126/scisignal.aai9192.

15. A. Kumari, J. Pineau, P.J. Sáez, M. Maurin, D. Lankar, M. San Roman, K. Hennig, V. F. Boura, R. Voituriez, M. C. I. Karlsson, M. Balland, A.-M. Lennon-Duménil, and P. Pierobon. Actomyosin-driven force patterning controls endocytosis at the immune synapse. Nature Communications, 10(1):1-14, 2019. ISSN 20411723. doi: 10.1038/s41467-019-10751-7. 
16. O. Thaunat, A. G. Granja, P. Barral, A. Filby, B. Montaner, L. Collinson, N. Martinez-Martin, N. E. Harwood, A. Bruckbauer, and F. D. Batista. Asymmetric segregation of polarized antigen on B cell division shapes presentation capacity. Science, 335(6067):457-479, 2012. ISSN 10959203. doi: 10.1126/science.1214100.

17. K. Pham, F. Sacirbegovic, and S. M. Russell. Polarized cells, polarized views: Asymmetric cell division in hematopoietic cells. Frontiers in Immunology, 5(FEB), 2014. ISSN 16643224. doi: 10.3389/fimmu.2014.00026.

18. Zhengpeng Wan, Xiangjun Chen, Haodong Chen, Qinghua Ji, Yingjia Chen, Jing Wang, Yiyun Cao, Fei Wang, Jizhong Lou, Zhuo Tang, and Wanli Liu. The activation of IgM- or isotype-switched IgG- and IgE-BCR exhibits distinct mechanical force sensitivity and threshold. eLife, 4(August):1-24, 2015. ISSN 2050-084X. doi: 10.7554/eLife.06925.

19. Hakim Boukellal, Otger Campás, Jean-François Joanny, Jacques Prost, and Cécile Sykes. Soft <i>Listeria</i>: Actin-based propulsion of liquid drops. Physical Review E, 69(6):061906, jun 2004. doi: 10.1103/PhysRevE.69.061906.

20. O. Campàs, T. Mammoto, S. Hasso, R. A. Sperling, O. Connell, A. G. Bischof, R. Maas, and D. A. Weitz. Embryonic Tissues. Nature Methods, 11(2):183-189, 2014. doi: 10.1038/nmeth.2761. Quantifying.

21. N. Bourouina, J. Husson, C. Hivroz, and N. Henry. Biomimetic droplets for artificial engagement of living cell surface receptors: The specific case of the T-cell. Langmuir, 28(14):6106-6113, 2012. ISSN 07437463. doi: 10.1021/la300398a.

22. K. Ben M'Barek, D. Molino, S. Quignard, M.-A. Plamont, Y. Chen, P. Chavrier, and J. Fattaccioli. Phagocytosis of immunoglobulin-coated emulsion droplets. Biomaterials, 51:270-277, 2015. ISSN 18785905. doi: 10.1016/j.biomaterials.2015.02.030.

23. L. Montel, L. Pinon, and J. Fattaccioli. A Multiparametric and High-Throughput Assay to Quantify the Influence of Target Size on Phagocytosis. Biophysical Journal, pages 1-12, 2019. ISSN 15420086. doi: 10.1016/j.bpj.2019.06.021.

24. B. Dumat, L. Montel, L. Pinon, P. Matton, L. Cattiaux, J. Fattaccioli, and J.-M. Mallet. Mannose-Coated Fluorescent Lipid Microparticles for Specific Cellular Targeting and Internalization via Glycoreceptor-Induced Phagocytosis. ACS Applied Bio Materials, 2019. ISSN 25766422. doi: 10.1021/acsabm.9b00793.

25. M. Vuica, S. Desiderio, and J. P. Schneck. Differential effects of B cell receptor and B cell receptor-Fc $\gamma$ RIIB1 engagement on docking of Csk to GTPase-activating protein (GAP)-associated p62. Journal of Experimental Medicine, 186(2):259-267, 1997. ISSN 00221007. doi: 10.1084/jem.186.2.259.

26. D. Lankar, H. Vincent-Schneider, V. Briken, T. Yokozeki, G. Raposo, and C. Bonnerot. Dynamics of major histocompatibility complex class II compartments during B cell receptor-mediated cell activation. Journal of Experimental Medicine, 195(4):461-472, 2002. ISSN 00221007. doi: 10.1084/jem.20011543.

27. Y. Zhang, A. McMullen, L.-L. Pontani, X. He, R. Sha, N. C. Seeman, J. Brujic, and P. M. Chaikin. Sequential self-assembly of DNA functionalized droplets. Nature Communications, 8(1):1-7, 2017. ISSN 20411723. doi: 10.1038/s41467-017-00070-0.

28. T. G. Mason and J. Bibette. Shear rupturing of droplets in complex fluids. Langmuir, 13(17):4600-4613, 1997. ISSN 07437463. doi: 10.1021/la9700580.

29. G. T. Vladisavljević, M. Shimizu, and T. Nakashima. Permeability of hydrophilic and hydrophobic Shirasu-porous-glass (SPG) membranes to pure liquids and its microstructure. Journal of Membrane Science, 250(1-2):69-77, 2005. ISSN 03767388. doi: 10.1016/j.memsci.2004.10.017.

30. L. Pinon, L. Montel, O. Mesdjian, M. Bernard, A. Michel, C. Ménager, and J. Fattaccioli. Kinetically Enhanced Fabrication of Homogeneous Biomimetic and Functional Emulsion Droplets. Langmuir, 34(50):15319-15326, dec 2018. ISSN 0743-7463. doi: 10.1021/acs.langmuir.8b02721.

31. N. Bufi, M. Saitakis, S. Dogniaux, A. Buschinger, O.and Bohineust, Al. Richert, M. Maurin, C. Hivroz, and A. Asnacios. Human primary immune cells exhibit distinct mechanical properties that are modified by inflammation. Biophysical Journal, 108(9):2181-2190, 2015. ISSN 15420086. doi: 10.1016/j.bpj.2015.03.047.

32. Michael Saitakis, Stéphanie Dogniaux, Christel Goudot, Nathalie Bufi, Sophie Asnacios, Mathieu Maurin, Clotilde Randriamampita, Atef Asnacios, and Claire Hivroz. Different TCR-induced T lymphocyte responses are potentiated by stiffness with variable sensitivityDifferent TCR-induced T lymphocyte responses are potentiated by stiffness with variable sensitivity. eLife, 6:1-29, 2017. ISSN 2050084X. doi: 10.7554/eLife.23190.

33. Pierre-Gilles de Gennes, Françoise Brochard-Wyart, and David Quéré. Capillarity and wetting phenomena. Springer, 2004. ISBN 9781441918338. doi: 10.1007/978-0-387-21656-0_9.

34. Phil Attard and Stan J. Miklavcic. Effective Spring Constant of Bubbles and Droplets. Langmuir, 17(26):8217-8223, dec 2001. ISSN 0743-7463. doi: 10.1021/la010969g.

35. A. Yeung, T. Dabros, and J. Masliyah. Does equilibrium interfacial tension depend on method of measurement? Journal of Colloid and Interface Science, $208(1): 241-247,1998$. ISSN 00219797. doi: $10.1006 /$ jcis. 1998.5807.

36. Daniel T.N. Chen, Qi Wen, Paul A. Janmey, John C. Crocker, and Arjun G. Yodh. Rheology of soft materials. Annual Review of Condensed Matter Physics, 1:301-322, 2010. ISSN 19475454. doi: 10.1146/annurev-conmatphys-070909-104120.

37. N. Desprat, A. Richert, J. Simeon, and A. Asnacios. Creep function of a single living cell. Biophysical Journal, 88(3):2224-2233, 2005. ISSN 00063495. doi: 10.1529/biophysj.104.050278.

38. Ben Fabry, Geoffrey N. Maksym, James P. Butler, Michael Glogauer, Daniel Navajas, and Jeffrey J. Fredberg. Scaling the microrheology of living cells. Physical Review Letters, 87(14):1-4, 2001. ISSN 00319007. doi: 10.1103/PhysRevLett.87.148102.

39. M. Balland, N. Desprat, D. Icard, S. Féréol, A. Asnacios, J. Browaeys, S. Hénon, and F. Gallet. Power laws in microrheology experiments on living cells: Comparative analysis and modeling. Physical Review E - Statistical, Nonlinear, and Soft Matter Physics, 74(2), 2006. ISSN 15502376. doi: 10.1103/PhysRevE.74.021911.

40. A. M. Skelley, O. Kirak, H. Suh, R. Jaenisch, and J. Voldman. Microfluidic control of cell pairing and fusion. Nature Methods, 6(2):147-152, 2009. ISSN 15487091. doi: 10.1038/nmeth.1290.

41. M. Jafarnejad, M. C. Woodruff, D. C. Zawieja, M. C. Carroll, and J. E. Moore. Modeling lymph flow and fluid exchange with blood vessels in lymph nodes. Lymphatic Research and Biology, 13(4): 234-247, 2015. ISSN 15578585. doi: 10.1089/lrb.2015.0028.

42. Fulvia Vascotto, Danielle Lankar, Pablo Vargas, Jheimmy Diaz, Delphine Le Roux, Delphine le Roux, Maria-isabel Yuseff, Maria-isabel Yuseff, Jean-Baptiste Sibarita, Marianne Boes, Graça Raposo, Evelyne Mougneau, Nicolas Glaichenhaus, Christian Bonnerot, Bénédicte Manoury, Ana-Maria Lennon-Duménil, Gabrielle Faure-André, Pablo Vargas, Jheimmy Diaz, Delphine le Roux, Maria-isabel Yuseff, Jean-Baptiste Sibarita, Marianne Boes, Graça Raposo, Evelyne Mougneau, Nicolas Glaichenhaus, Christian Bonnerot, Bénédicte Manoury, and Ana-Maria Lennon-Duménil. The actin-based motor protein myosin II regulates MHC class II trafficking and BCR-driven antigen presentation. Journal of Cell Biology, 176(7):1007-1019, mar 2007. ISSN 0021-9525. doi: 
$10.1083 /$ jcb.200611147.

43. Jia C. Wang, Jeff Y.J. Lee, Sonja Christian, May Dang-Lawson, Caitlin Pritchard, Spencer A. Freeman, and Michael R. Gold. The Rap1-cofilin-1 pathway coordinates actin reorganization and MTOC polarization at the B cell immune synapse. Journal of Cell Science, 130(6):1094-1109, 2017. ISSN 14779137. doi: 10.1242/jcs.191858.

44. Mukund Gupta, Bibhu Ranjan Sarangi, Joran Deschamps, Yasaman Nematbakhsh, Andrew Callan-Jones, Felix Margadant, René-Marc Mège, Chwee Teck Lim, Raphaël Voituriez, and Benoît Ladoux. Adaptive rheology and ordering of cell cytoskeleton govern matrix rigidity sensing. Nature communications, 6(May):7525, 2015. ISSN 2041-1723. doi: 10.1038/ncomms8525.

45. D. Mitrossilis, J. Fouchard, D. Pereira, F. Postic, A. Richert, M. Saint-Jean, and A. Asnacios. Real-time single-cell response to stiffness. Proceedings of the National Academy of Sciences of the United States of America, 107(38):16518-16523, 2010. ISSN 10916490. doi: 10.1073/pnas.1007940107.

46. J. Husson, K. Chemin, A. Bohineust, C. Hivroz, and N. Henry. Force generation upon T cell receptor engagement. PLoS ONE, 6(5), 2011. ISSN 19326203. doi: 10.1371/journal.pone.0019680.

47. Sara V. Merino-Cortés, Sofia R. Gardeta, Sara Roman-Garcia, Ana Martínez-Riaño, Judith Pineau, Rosa Liebana, Isabel Merida, Ana Maria Lennon Dumenil, Paolo Pierobon, Julien Husson, Balbino Alarcon, and Yolanda R. Carrasco. Diacylglycerol kinase $\zeta$ promotes actin cytoskeleton remodeling and mechanical forces at the B cell immune synapse. Science Signaling, 13(627), apr 2020. ISSN 19379145. doi: 10.1126/scisignal.aaw8214.

48. N. E. Harwood and F. D. Batista. Early Events in B Cell Activation. Annual Review of Immunology, 28(1):185-210, 2010. ISSN 0732-0582. doi: 10.1146/annurev-immunol-030409-101216.

49. B. Treanor, D. Depoil, A. Bruckbauer, and F. D. Batista. Dynamic cortical actin remodeling by ERM proteins controls BCR microcluster organization and integrity. Journal of Experimental Medicine, 208(5):1055-1068, 2011. ISSN 00221007. doi: 10.1084/jem.20101125.

50. C. Guedj, N. Abraham, D. Jullié, and C. Randriamampita. T cell adhesion triggers an early signaling pole distal to the immune synapse. Journal of Cell Science, 129(13):2526-2537, 2016. ISSN 14779137. doi: $10.1242 /$ jcs. 182311 .

51. Jason Yi, Xufeng Wu, Andrew H. Chung, James K. Chen, Tarun M. Kapoor, and John A. Hammer III. Centrosome repositioning in T cells is biphasic and driven by microtubule end-on captureshrinkage. Journal of Cell Biology, 202(5):779-792, 2013. ISSN 00219525. doi: 10.1083/jcb.201301004.

52. N. Bourouina, J. Husson, F. Waharte, R. B. Pansu, and N. Henry. Formation of specific receptor-ligand bonds between liquid interfaces. Soft Matter, 7(19):9130-9139, 2011. ISSN 1744683X. doi: $10.1039 / \mathrm{c} 1 \mathrm{sm} 05659 \mathrm{j}$.

53. R. Basu and M. Huse. Mechanical Communication at the Immunological Synapse. Trends in Cell Biology, 27(4):241-254, 2017. ISSN 18793088. doi: 10.1016/j.tcb.2016.10.005.

54. R. Wang, S. Zheng, and Y. George Zheng. Polymer Matrix Composites and Technology. Woodhead Publishing, 1st edition, 2011. ISBN 978-0-85709-221-2,978-0-85709-222-9.

55. D. Vorselen, Y. Wang, M. M. de Jesus, P. K. Shah, M. J. Footer, M. Huse, W. Cai, and J. A. Theriot. Microparticle traction force microscopy reveals subcellular force exertion patterns in immune cell-target interactions. Nature Communications, 11(1), 2020. ISSN 20411723. doi: 10.1038/s41467-019-13804-z.

56. A. Yeung, T. Dabros, J. Masliyah, and J. Czarnecki. Micropipette: A new technique in emulsion research. Colloids and Surfaces A: Physicochemical and Engineering Aspects, 174(1-2):169-181, 2000. ISSN 09277757. doi: 10.1016/S0927-7757(00)00509-4.

57. N. Desprat, A. Guiroy, and A. Asnacios. Microplates-based rheometer for a single living cell. Review of Scientific Instruments, 77:1-9, 2006.

58. Johannes Schindelin, Ignacio Arganda-Carreras, Erwin Frise, Verena Kaynig, Mark Longair, Tobias Pietzsch, Stephan Preibisch, Curtis Rueden, Stephan Saalfeld, Benjamin Schmid, Jean Yves Tinevez, Daniel James White, Volker Hartenstein, Kevin Eliceiri, Pavel Tomancak, and Albert Cardona. Fiji: An open-source platform for biological-image analysis. Nature Methods, 9(7):676-682, 2012. ISSN 15487091. doi: 10.1038/nmeth.2019.

59. O. Mesdjian, N. Ruyssen, M. C. Jullien, R. Allena, and J. Fattaccioli. Enhancing the capture efficiency and homogeneity of single-layer flow-through trapping microfluidic devices using oblique hydrodynamic streams. Microfluidics and Nanofluidics 2021 25:11, 25(11):1-10, oct 2021. ISSN 1613-4990. doi: 10.1007/S10404-021-02492-1.

60. Daniel S. Banks and Cécile Fradin. Anomalous diffusion of proteins due to molecular crowding. Biophysical journal, 89(5):2960-2971, 2005. ISSN 0006-3495. doi: 10.1529/BIOPHYSJ.104.051078.

61. T. C. Werner, J. R. Bunting, and R. E. Cathou. The shape of immunoglobulin G molecules in solution. Proceedings of the National Academy of Sciences of the United States of America, 69(4): 795-799, 1972. ISSN 00278424. doi: 10.1073/pnas.69.4.795.

62. P. Durand-Smet, E. Gauquelin, N. Chastrette, A. Boudaoud, and A. Asnacios. Estimation of turgor pressure through comparison between single plant cell and pressurized shell mechanics. Physical Biology, 14(5), 2017. ISSN 14783975. doi: 10.1088/1478-3975/aa7f30.

63. Donald Wlodkowic, Shannon Faley, Michele Zagnoni, John P. Wikswo, and Jonathan M. Cooper. Microfluidic Single Cell Array Cytometry for the Analysis of Tumour Apoptosis. Analytical Chemistry, 81(13), 2009. ISSN 15378276. doi: 10.1021/ac9008463.Microfluidic.

64. Natalija Backmann, Natascha Kappeler, Thomas Braun, François Huber, Hans Peter Lang, Christoph Gerber, and Roderick Y.H. Lim. Sensing surface PEGylation with microcantilevers. Beilstein Journal of Nanotechnology, 1(1):3-13, 2010. ISSN 21904286. doi: 10.3762/bjnano.1.2.

65. Anna Labernadie, Anaïs Bouissou, Patrick Delobelle, Stéphanie Balor, Raphael Voituriez, Amsha Proag, Isabelle Fourquaux, Christophe Thibault, Christophe Vieu, Renaud Poincloux, Guillaume M. Charrière, and Isabelle Maridonneau-Parini. Protrusion force microscopy reveals oscillatory force generation and mechanosensing activity of human macrophage podosomes. Nature Communications, 5, 2014. ISSN 20411723. doi: 10.1038/ncomms6343. 